\title{
PraxisWissen
}

EIN SERVICE DER KASSENÄRZTLICHEN BUNDESVEREINIGUNG

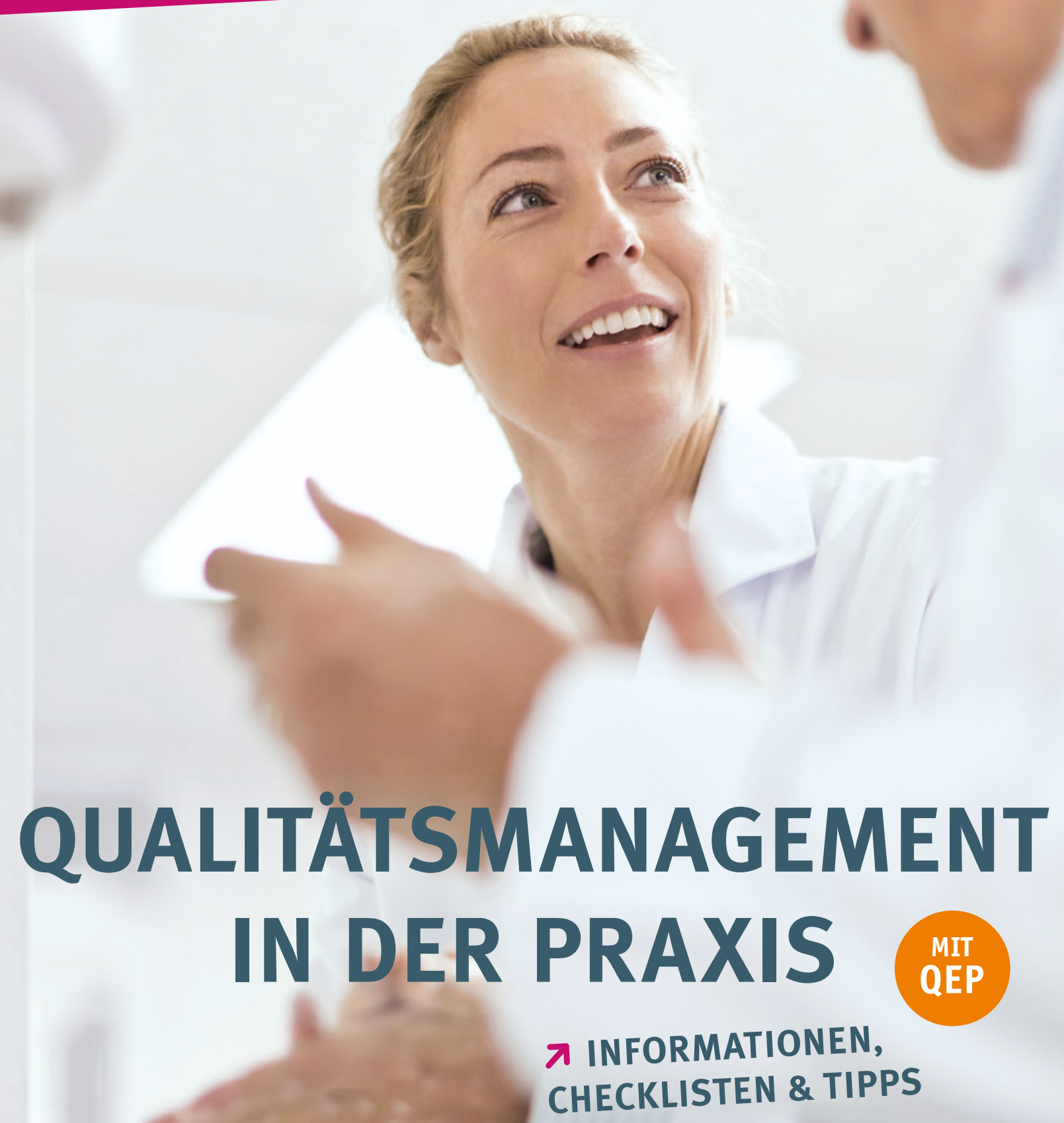




\section{LIEBE KOLLEGINNEN, LIEBE KOLLEGEN,}

als niedergelassene Ärzte und Psychotherapeuten garantieren Sie Ihren Patienten eine ambulante Versorgung auf höchstem Niveau. Dies stellt täglich hohe Anforderungen an Sie und Ihr Praxisteam.

Qualitätsmanagement kann dabei vieles im Praxisalltag erleichtern. Qualitätsmanagement bedeutet, alle fachlichen und organisatorischen Aktivitäten, die zu einer guten Versorgung beitragen, zu bündeln und zu systematisieren. Die Abläufe sind so organisiert, dass alles reibungslos funktioniert. Qualitätsmanagement eröffnet Gestaltungsspielräume und bewahrt Autonomie: Fragen der Qualität in den eigenen Händen zu behalten und dafür Verantwortung zu übernehmen, unterstreicht die ärztliche und psychotherapeutische Freiberuflichkeit.

Auf den folgenden Seiten finden Sie praktische Tipps zur Umsetzung, kurze Checklisten und Hinweise auf weiterführende Informationen und Unterstützungsangebote. Entwickeln Sie mit einem Qualitätsmanagement Ihre Praxis oder Ihr MVZ kontinuierlich weiter. Nicht zuletzt können Sie damit Ihre gute Qualität sichtbar machen.

IHRE KASSENÄRZTLICHE BUNDESVEREINIGUNG

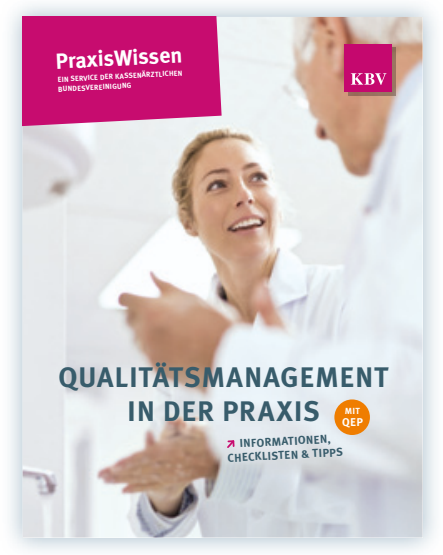

INHALT

Qualität in der Praxis Seite 1

Qualitätsmanagement: Darum geht es Seite 2

Reportage: QM in einer onkologischen Praxis Seite 4

Qualitätsmanagement: So funktioniert es Seite 6

Fokus: QEP - aus der Praxis für die Praxis Seite 6

QM unterstützt: Praxisführung

und Praxisorganisation Seite 8

QM sorgt für Sicherheit: Patientenversorgung Seite 12

Fokus: Fortbildung Seite 16

Interview: Dr. Andreas Gassen Seite 17

QM: Tipps für Einsteiger Seite 18

Bericht: QM für neu Niedergelassene Seite 19

QM in der Psychotherapie Seite 20

Interview: Dipl.-Psych. Julia Bellabarba Seite 20

Auf einen Blick: Das bringt QM Seite 21 


\section{Qualität in der Praxis}

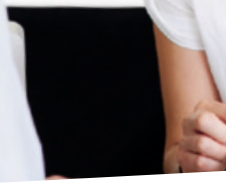

Qualität ist kein Zufall: Für Ärzte und Psychotherapeuten ist es seit jeher selbstverständlich, ihr Handeln stets zu hinterfragen und an fachlichen Standards auszurichten. So gibt es in der ambulanten Versorgung von gesetzlich krankenversicherten Patienten eine Fülle von Regeln und Vorgaben, die eine hohe Qualität der Behandlung sichern, fördern und kontinuierlich verbessern. Das beginnt schon bei der Zulassung als Vertragsarzt oder Vertragspsychotherapeut, die eine abgeschlossene Facharztausbildung beziehungsweise eine abgeschlossene Ausbildung zum Psychologischen Psychotherapeuten erfordert. Danach dürfen sie viele Leistungen nur durchführen und abrechnen, wenn sie zusätzliche Qualitätsanforderungen erfüllen. Dieses hohe Niveau zu halten und weiterzuentwickeln, ist eine der Hauptaufgaben der Kassenärztlichen Vereinigungen und der KBV.

\section{VERSORGUNG AUF HÖCHSTEM NIVEAU}

Viele Untersuchungs- und Behandlungsverfahren in der ambulanten Versorgung unterliegen speziellen Qualitätsanforderungen. So müssen Ärzte, die bestimmte ambulante Operationen oder Darmspiegelungen durchführen, regelmäßig ihre Qualifikation nachweisen. Neben dem fachlichen Wissen werden auch die Geräte und die Hygiene in den Praxen geprüft und gegebenenfalls Verbesserungsvorschläge gemacht. Nur wenn die Qualität stimmt, dürfen die Ärzte diese besonders qualitätsgesicherten Leistungen durchführen. Für die Qualitätssicherung gelten bundesweit dieselben Maßstäbe. Das hilft Ärzten und Psychotherapeuten dabei, ihre Patienten stets nach aktuellen fachlichen Standards und neuesten wissenschaftlichen Erkenntnissen zu versorgen.

\section{QUALITÄT FÖRDERN UND GESTALTEN}

Auch die Praxisorganisation unterliegt klaren Regeln: Ein individuell auf die Praxis, das MVZ oder das Ärztenetz abgestimmtes Qualitätsmanagement (QM) hilft dabei, die Qualität medizinischer Leistungen zu erhöhen und Abläufe effizienter zu gestalten. Das reicht von der Optimierung der Terminplanung über die strukturierte Durchführung von Untersuchungen bis zur Erstellung und Überprüfung des Notfallplans. Dabei profitieren vom Qualitätsmanagement nicht nur die Patienten, sondern auch das Team und nicht zuletzt die Praxisleitung: Transparente Strukturen und klare Zuständigkeiten erleichtern das Handeln. Sie sorgen für einen funktionierenden Arbeitsalltag und eine hohe Arbeitszufriedenheit.

\section{CHANCE FÜR DIE PRAXIS}

Zum Qualitätsmanagement sind Ärzte und Psychotherapeuten verpflichtet. Doch es ist mehr als eine gesetzliche Forderung: Es bietet Ärzten und Psychotherapeuten die Chance, bestehende Strukturen und Abläufe zu analysieren und kontinuierlich zu verbessern. Denn nur wer seine Stärken und Schwächen kennt, kann sich weiterentwickeln. Freiwillige Qualitätsinitiativen wie die Teilnahme an Qualitätszirkeln oder Peer-Review-Verfahren spielen dabei neben den gesetzlichen Vorgaben ebenfalls eine große Rolle. Die grundlegenden Anforderungen an ein einrichtungsinternes Qualitätsmanagement hat der Gemeinsame Bundesausschuss in einer Richtlinie geregelt. 


\section{Qualitätsmanagement: Darum geht es}

Was erst einmal kompliziert klingt, ist eigentlich ganz

einfach: Beim Qualitätsmanagement geht es um die

Gestaltung der fachlichen und organisatorischen Abläufe. Viele

Instrumente wenden Ärzte und Psychotherapeuten - ob bewusst

oder unbewusst - dabei schon immer an. Dies systematisch und

kontinuierlich zu tun, erleichtert die Arbeit und trägt zum Erfolg

einer Praxis oder eines Medizinischen Versorgungszentrums bei.

\section{EIN INSTRUMENT DER UNTERNEHMENSFÜHRUNG}

Qualitätsmanagement unterstützt die Praxisleitung und das Team dabei, Arbeitsabläufe zu strukturieren, Verantwortlichkeiten festzulegen und Risiken frühzeitig zu erkennen. Ziel ist es, alle Tätigkeiten konsequent an fachlichen, gesetzlichen und vertraglichen Grundlagen auszurichten - und sich dabei möglichst nah an den Bedürfnissen von Patienten und Mitarbeitern zu orientieren. So kann für alle eine größtmögliche Sicherheit und Zufriedenheit erreicht werden. Konkret bedeutet das: das eigene Tun beobachten, gegebenenfalls dokumentieren, und, wo Verbesserungen möglich und sinnvoll sind, verändern.

\section{OHNE QM GEHT'S NICHT}

Ob gute Praxisorganisation nun Qualitätsmanagement oder gesunder Menschenverstand genannt wird, ist gleich - ohne geht es nicht. Damit die bewährten Methoden aus dem Werkzeugkasten der Unternehmensführung auch von allen an der vertragsärztlichen Versorgung teilnehmenden Ärzten, Psychotherapeuten und MVZ genutzt werden, sind sie seit 2004 gesetzlich verpflichtet, ein sogenanntes einrichtungsinternes Qualitätsmanagement einzuführen und weiterzuentwickeln ( $\$ 135$ a Sozialgesetzbuch V). In der QM-Richtlinie des Gemeinsamen Bundesausschusses (G-BA) sind die Ziele, Grundsätze, Instrumente und der Zeitrahmen für eine Einführung und Weiterentwicklung festgelegt.

\section{STICHPROBENERGEBNISSE ZUR QM-RICHTLINIE IN 2014}

Die Stichprobe zur QM-Richtlinie in 2014 zeigt, dass etwa 80 Prozent aller befragten Ärzte und Psychotherapeuten die Anforderungen der Richtlinie zeitgerecht erfüllen, viele davon sogar schneller als vorgegeben. Etwa 20 Prozent der Praxen und MVZ müssen die in der Richtlinie geforderten Instrumente noch systematischer anwenden und Maßnahmen gegebenenfalls auch zeitnaher umsetzen.

\section{DIE QM-RICHTLINIE}

$\lambda$ zielt auf größtmögliche

Patientensicherheit und eine bewusste Patientenorientierung.

$\lambda$ fordert, dass der Aufwand für QM in einem angemessenen Verhältnis zur personellen und strukturellen Ausstattung der Praxis oder des MVZ stehen soll.

$\pi$ beschreibt die grundsätzlichen Anforderungen für eine erfolgreiche Einführung und Umsetzung von QM.

$\nearrow$ gibt die Ziele des Qualitätsmanagements vor.

$\pi$ führt Methoden wie das Festlegen von Verantwortlichkeiten, Prozessund Ablaufbeschreibungen und Schnittstellenmanagement auf.

$\pi$ gibt konkrete Instrumente wie regelmäßige Teamsitzungen,

Patienten- und Mitarbeiterbefragungen, Regelungen zum Umgang mit Risiken, sicherheitsrelevanten Ereignissen und Fehlern vor.

$\pi$ fordert zum Beispiel ein Hygieneund ein Notfallmanagement sowie Festlegungen zur sicheren Arzneimitteltherapie. 


\section{FÖRDERGELDER FÜR DIE EINFÜHRUNG UND WEITERENTWICKLUNG VON QM}

\section{Manchmal ist es hilfreich eine externe Beratung in Anspruch zu nehmen, denn} eine Sicht von außen kann sinnvoll sein. Für Kosten, die dafür entstehen, können Sie eine Förderung des Bundesamts für Wirtschaft und Ausfuhrkontrolle beantragen. Es werden teilweise bis zu 50 Prozent der Beratungskosten erstattet. Weitere Informationen dazu unter www.beratungsfoerderung.info

\section{DER ERSTE SCHRITT: STANDORTBESTIMMUNG}

QM ist Teamarbeit: Bei der Einführung eines Qualitätsmanagement-Systems sollten möglichst alle in der Praxis mit eingebunden werden. Die Leitung muss voll und ganz hinter den vereinbarten Aktivitäten und Maßnahmen stehen. Sich gemeinsam alle Bereiche der Praxis oder des Medizinischen Versorgungszentrums näher anzuschauen, die von einer Verbesserung profitieren können, ist ein guter Ausgangspunkt für die Einführung des Qualitätsmanagements - die sogenannte Selbstbewertung oder Standortbestimmung. Dabei kann eine Checkliste helfen (siehe rechts).

\section{TIPP}

Schätzen Sie selbst ein, inwieweit Sie die in der QM-Richtlinie geforderten Instrumente bereits in Ihrem Praxisalltag umgesetzt haben. Bewerten Sie Ihr Qualitätsmanagement und leiten Sie daraus Ziele $\mathrm{ab}$, was vielleicht noch verbessert werden kann. Sie finden in diesem Heft zu allen relevanten QM-Instrumenten praktische Tipps und Hinweise auf weiterführende Informationen.

\section{DIE QM-STANDORTBESTIMMUNG}

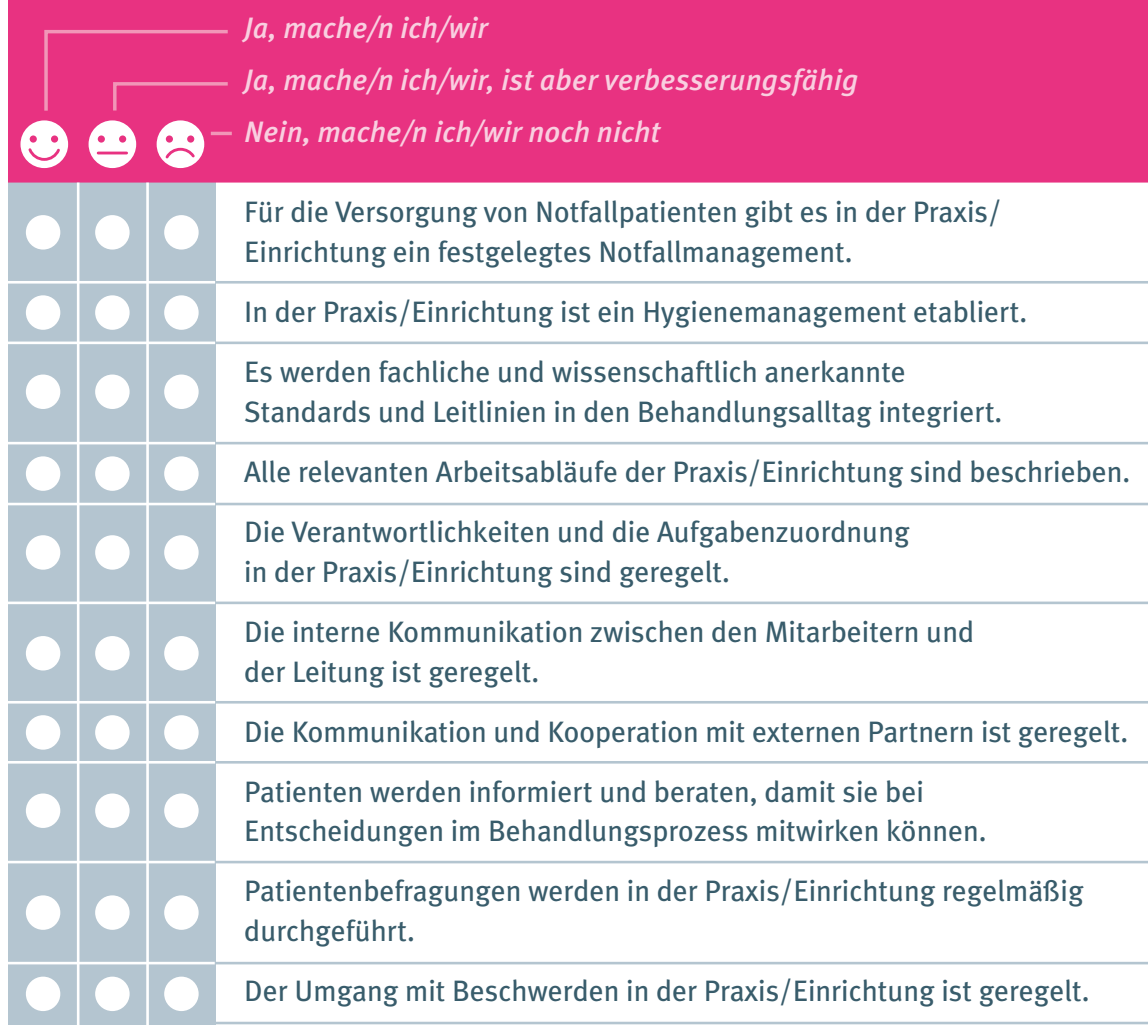

Ein Selbstbewertungsbogen, der alle Anforderungen der QM-Richtlinie beinhaltet, steht auf der Webseite der KBV zur Verfügung.

MEHR INFORMATIONEN

त QM-Richtlinie des G-BA: www.g-ba.de

入 Kurzinformation zur „QM-Richtlinie vertragsärztliche Versorgung“: www.kbv.de, Suchbegriff „Gesetzliche Vorgaben zu QM“

$\pi$ Selbstbewertungsbogen:

www.kbv.de, Suchbegriff „Selbstbewertungsbogen“ 


\section{EXAKTE ARBEITSPLATZBESCHREIBUNG FÜR MEHR VERBINDLICHKEIT}

Exakte Arbeitsplatzbeschreibungen und Terminvorgaben machen Arbeitsanweisungen leichter und führen zu mehr Verbindlichkeit, weil sie von jedem verstanden werden. Die Ärzte müssen sich - wie überall - darauf verlassen können, dass ihre Anordnungen vom Personal korrekt umgesetzt werden, ganz gleich ob an der Rezeption, im Labor oder in der Tagesklinik. „Und das spüren auch die Patienten“, ergänzt Sabine Kulawick und erläutert: „Viele Patienten sind aufgrund ihrer Erkrankung und Therapiepläne regelmäßig bei uns. Sie bekommen ziemlich gut mit, wie souverän wir uns organisiert haben, wie gewissenhaft wir die einzelnen Arbeitsschritte ausführen und ob wir ein Team sind, dem sie vertrauen können." Wöchentliche Besprechungen, in denen es nicht nur um Termine und aktuelle Aufgaben geht, sondern auch über Probleme gespro- chen wird, sollen zu jenem offenen Klima untereinander beitragen, in dem Fehler vorbehaltlos und angstfrei zur Sprache kommen können.

\section{PATIENTEN VERSTÄNDLICH INFORMIEREN}

Auch die Ärztinnen und Ärzte müssen sich untereinander immer wieder vergewissern, dass sie in allen wichtigen Fragen des Praxisprofils, der Umsetzung der Behandlungsleitlinien, der gemeinsam besprochenen Therapien usw. „ähnlich denken und handeln", wie Dr. Sauer es nennt. Auch bei ihnen sind scheinbare Selbstverständlichkeiten Gegenstand des Qualitätsmanagements, wie zum Beispiel das ärztliche Aufklärungsgespräch. Nicht selten entscheidet der erste Arzt-Patienten-Kontakt über das so notwendige gegenseitige Vertrauen in die Diagnostik und Therapie. Für Dr. Sauer ist deswegen wichtig: „Unsere Patienten müssen verständlich über alles aufgeklärt werden, was zu ihrem Krankheitsbild und zur Therapie gehört. Wir müssen geduldig auf ihre Ängste, Zweifel und Fragen eingehen. Das haben wir für uns ganz verbindlich geregelt."

Ein gutes Praxismanagement kann man spüren, das ist die beste Mundpropaganda, sind sich Dr. Sauer und Frau Kulawick einig. Und für Letzteres sorgen die Ärzte und Mitarbeiter auf ihre Weise - jenseits aller QualitätsmanagementHandbücher: Nach Feierabend und an so manchem freien Wochenende unterstützen sie den örtlichen, von ihnen selbst initiierten Verein für krebskranke Patienten, organisieren Patientenveranstaltungen und verleihen ihrem Teamgeist auch durch einen eigenen Praxischor Nachdruck, der in Potsdam schlicht unter „MVZ-Chor“ firmiert.

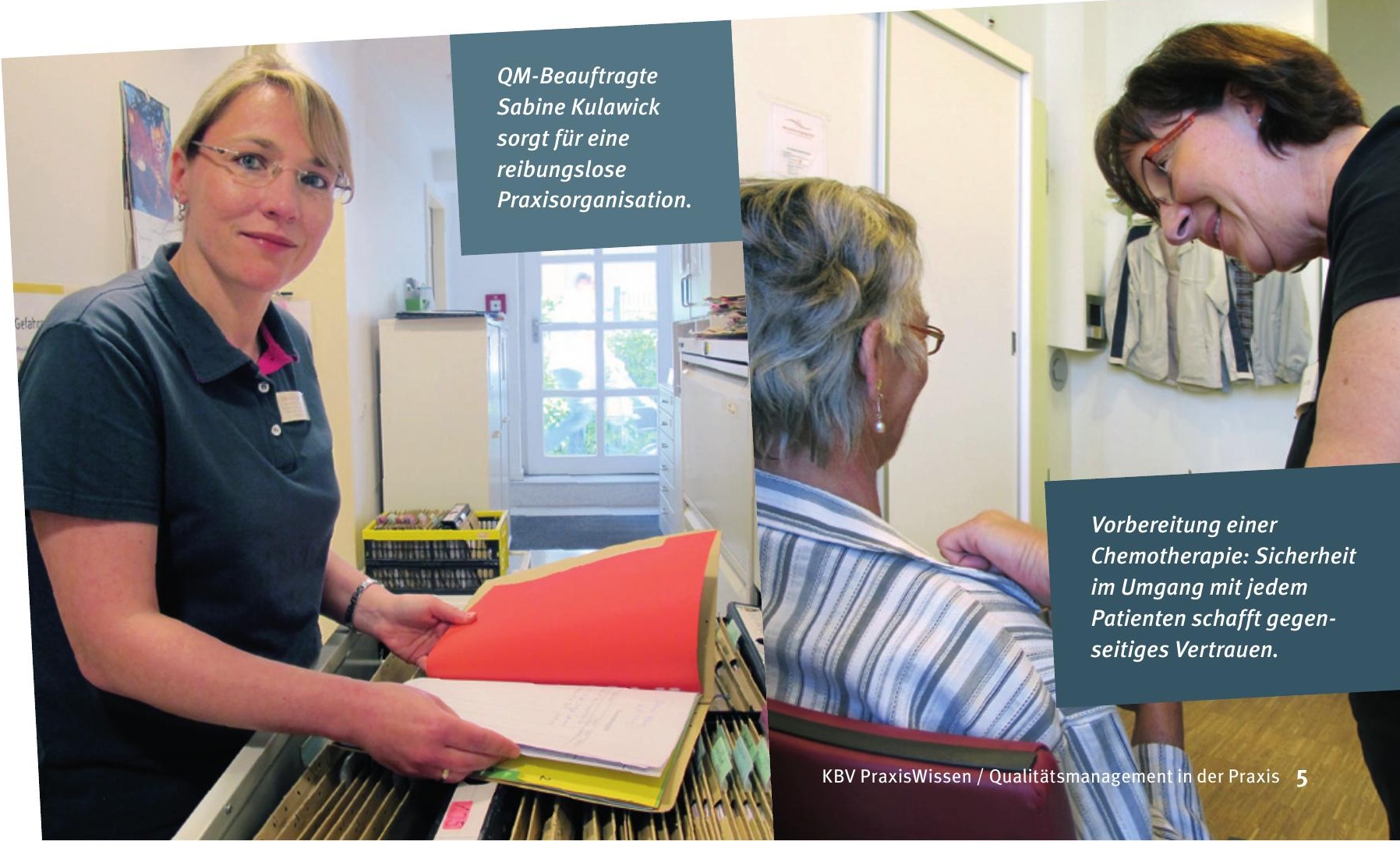




\section{Qualitätsmanagement: So funktioniert es}

\section{QEP - AUS DER PRAXIS FÜR DIE PRAXIS}

Nach einer Selbstbewertung der Praxis können Ärzte und Psychotherapeuten direkt mit der Umsetzung loslegen und konkrete Ziele und Aufgaben festlegen. Welche Abläufe zum Beispiel sollten wie optimiert werden, welche Schwachstellen in der Praxisorganisation wie behoben werden? Was daher nicht fehlen darf, ist ein Zeit- und Maßnahmenplan. Es sollte im Team klar festgelegt werden, wer welche dieser Aufgaben bis wann übernimmt. Anschließend geht es an die Umsetzung. Das Erreichte wird dann anhand der selbst gesetzten Ziele regelmäßig überprüft und kontinuierlich weiterentwickelt.

\section{SO GEHT'S: PLAN-DO-CHECK-ACT}

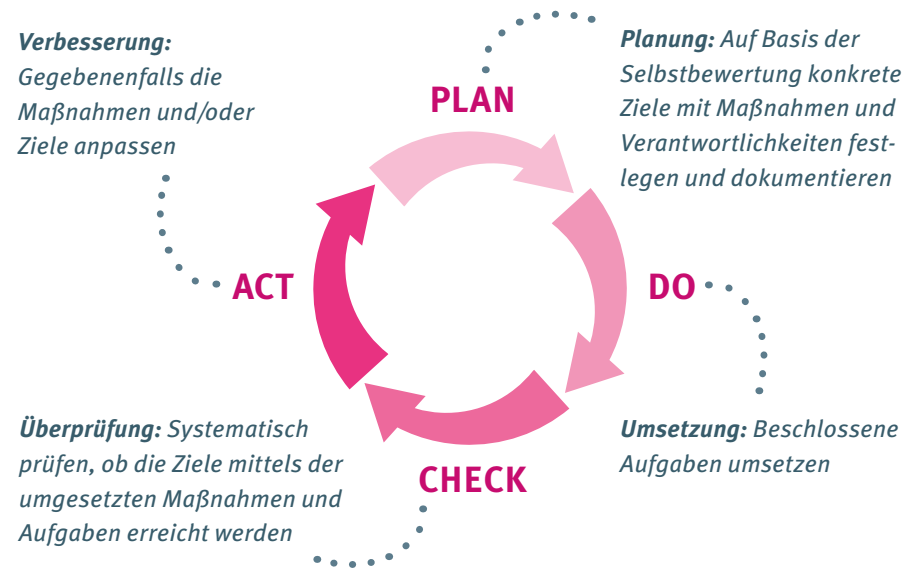

\section{QM-VERFAHREN HELFEN BEI DER UMSETZUNG}

Beim Aufbau eines internen QM-Systems helfen sogenannte Qualitätsmanagement-Verfahren wie $\mathrm{QEP}^{\circledast}, \mathrm{KTQ}^{\circledR}$ oder EPA. Auch verschiedene regionale Einstiegsangebote unterstützen beim Start mit QM: KPQM, qu.no, QisA ${ }^{\circledast}$. Die Normen der ISO (Internationale Organisation für Normung), in denen auch Anforderungen an QualitätsmanagementSysteme festgelegt sind, zum Beispiel DIN EN ISO 9001, gelten branchenübergreifend. Etwa ein Drittel der Praxen nutzen QEP - Qualität und Entwicklung in Praxen ${ }^{\circledast}$. Für die Einführung von Qualitätsmanagement ist jedoch kein bestimmtes System gesetzlich vorgeschrieben. Eine Übersicht über verschiedene Qualitätsmanagement-Verfahren finden Sie unter www.kbv.de, Suchbegriff "QM-Verfahren“"
QEP steht für Qualität und Entwicklung in Praxen. Das Qualitätsmanagement-Verfahren wurde von der KBV und den Kassenärztlichen Vereinigungen als Serviceangebot eigens für die Niedergelassenen entwickelt, um sie bei der Einführung eines internen Qualitätsmanagements zu unterstützen. QEP bietet für alle praxisrelevanten Themen Qualitätsziele und konkrete Vorschläge zur Umsetzung an. QEP ist spezifisch auf die Abläufe und Bedingungen in der Praxis zugeschnitten und kann individuell an die jeweilige Fachrichtung, Praxisgröße und Organisationsform angepasst werden. Es besteht aus verschiedenen Bausteinen, die aufeinander abgestimmt, kombinierbar und schrittweise umsetzbar sind. Ein Team aus Ärzten, Psychotherapeuten, Praxismitarbeitern und QM-Experten war an der Entwicklung beteiligt.

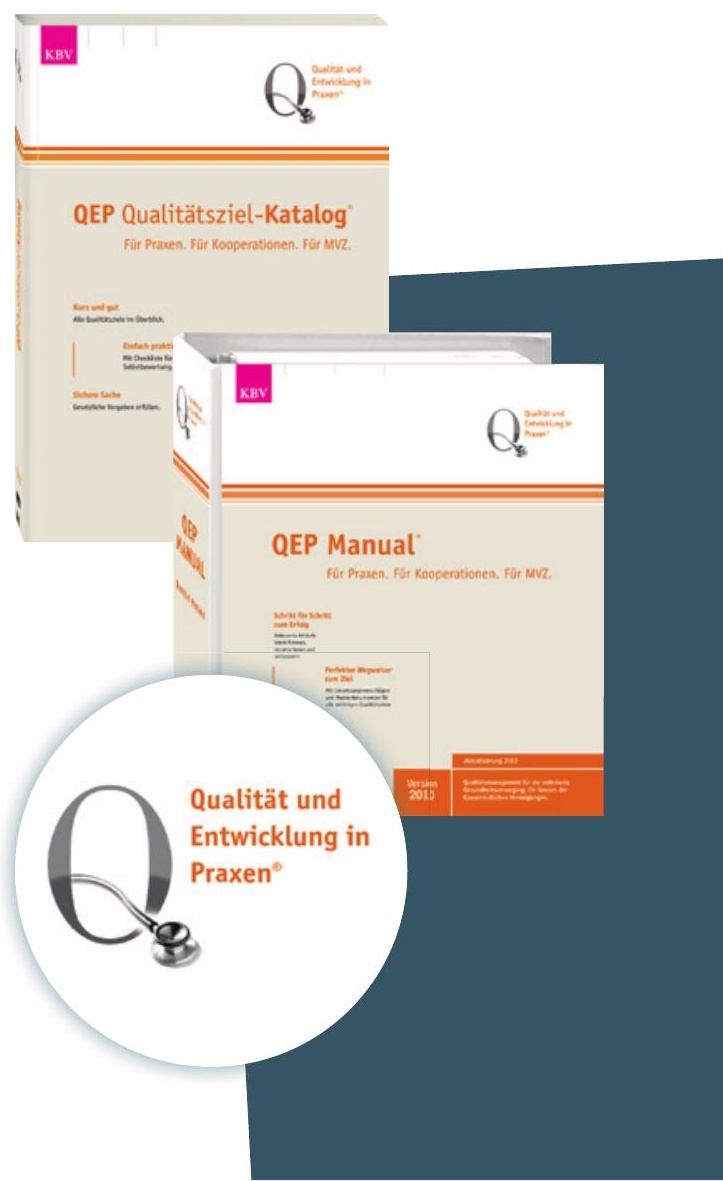




\section{QEP-QUALITÄTSZIEL-KATALOG ${ }^{\circledR}$ : SCHRITT FÜR SCHRITT ZU EINEM PASSGENAUEN QM}

Das Herzstück von QEP ist der QualitätszielKatalog. Mit ihm lassen sich gesetzliche Verpflichtungen und Vorgaben leicht auf die eigene Praxis oder das MVZ übertragen und umsetzen. Der Katalog vermittelt einen guten thematischen Überblick, beschreibt die Qualitätsziele und gibt zahlreiche Anregungen und Ideen, was zu tun und gegebenenfalls zu verbessern ist. Für jede Praxis sind die Qualitätsziele je nach Leistungsspektrum unterschiedlich relevant. Die hervorgehobenen Kernziele machen es leicht, die besonders wichtigen Aufgaben von denen zu unterscheiden, die auch zu einem späteren Zeitpunkt umgesetzt werden können. Die gesetzlichen Bestimmungen sind im Katalog besonders markiert.

\section{Die Kapitel}

\begin{tabular}{ll}
\hline 1 & Patientenversorgung \\
\hline 2 & Patientenrechte \& Patientensicherheit \\
\hline 3 & Mitarbeiter \& Fortbildung \\
\hline 4 & Führung \& Organisation \\
\hline 5 & Qualitätsentwicklung \\
\hline
\end{tabular}

\section{QEP-MANUAL ${ }^{\circledR}$ : UMSETZUNGSVORSCHLÄGE UND ZAHLREICHE MUSTERDOKUMENTE}

In Ergänzung zum QEP-Qualitätsziel-Katalog bietet das QEP-Manual viele praktische Tipps und Erläuterungen, Vorschläge zum Praxismanagement und Hinweise auf weiterführende Informationen. Das Manual enthält darüber hinaus zahlreiche Musterdokumente - beispielsweise für Checklisten, Ablaufbeschreibungen, Protokolle, Interne Regelungen und Patienteninformationen, die auch für die Erstellung eines eigenen Praxishandbuchs verwendet werden können. Qualitätsziel-Katalog und Manual helfen dabei, kritische Abläufe zu identifizieren, Risiken sowie mögliche Fehlerquellen aufzuspüren und Konsequenzen daraus abzuleiten.

\section{QEP-ZERTIFIZIERUNG: QUALITÄT SICHTBAR MACHEN}

Neben den verschiedenen Bausteinen für den Aufbau eines praxisorientierten QMSystems bietet QEP die Möglichkeit, die Umsetzung in der Praxis durch einen neutralen Dritten prüfen zu lassen. Über 1.500
Praxen haben sich bislang freiwillig nach QEP zertifizieren lassen und können damit ihr hohes Engagement nach außen darlegen.

\section{TIPP}

\section{QEP-EINFÜHRUNGSSEMINAR}

Für einen Einstieg ins Qualitätsmanagement bietet sich der Besuch eines QEP-Einführungsseminars an. Es ist empfehlenswert, dass sowohl die Praxisleitung als auch ein Mitarbeiter aus dem Team daran teilnehmen. In dem Seminar lernen die Teilnehmer den QEP-Qualitätsziel-Katalog kennen und testen das Arbeiten mit dem QEP-Manual. Sie erfahren auch, welche Schritte für eine freiwillige QEP-Zertifizierung (sie ist gesetzlich nicht vorgeschrieben) erforderlich sind.

\author{
MEHR INFORMATIONEN \\ $\pi$ KBV-Themenseite QEP: \\ www.kbv.de/qep \\ $\pi$ Abonnieren Sie den QEP-Newsletter: \\ www.kbv.de, Suchbegriff \\ "QEP-Newsletter"
}

\section{TYPISCH FÜR QEP}

$\lambda$ Das Konzept ist wissenschaftlich evaluiert, gute Verständlichkeit und Anwendbarkeit sowie praktische Relevanz sind belegt. $\lambda$ Die Patientenversorgung steht im Mittelpunkt.

$\pi$ Das Instrumentarium ist leicht umsetzbar sowie kostengünstig.

$\lambda$ Der Aufbau eines QM-Systems kann mit QEP schrittweise und individuell erfolgen.

$\lambda$ Begleitende Unterstützungsangebote stehen zur Verfügung.

$\lambda$ Die Anforderungen sind als Nachweise/Indikatoren formuliert, dadurch transparent und messbar.

$\lambda$ Eine spätere Zertifizierung ist möglich.

Wie QEP in der Praxis umgesetzt werden kann, erfahren Sie auch auf www.kv-on.de. Hier werden die einzelnen Kapitel in kurzen Videos anschaulich vorgestellt.

\section{BEZUGSQUELLEN UND WEITERE INFOS}

QEP-Einführungsseminare bieten die meisten Kassenärztlichen Vereinigungen und einige Berufsverbände an (Kosten: ca. 150 bis 200 Euro). Weitere Informationen auf der Webseite der KBV, www.kbv.de, Suchbegriff „QEP-Einführungsseminar

Der QEP-Qualitätsziel-Katalog (29,95 Euro) und das QEPManual (199 Euro) sind im Deutschen Ärzte-Verlag erschienen und können dort über www.aerzteverlag.de, Rubrik „Shop“ oder über den Buchhandel bezogen werden.

In Relation zu vergleichbaren Angeboten kommerzieller Anbieter bewegen sich diese Kosten deutlich im unteren Bereich. Und: All diese Investitionen lohnen sich, denn ein erfolgreiches Qualitätsmanagement hilft auch, Kosten zu sparen. 


\section{QM unterstützt: Praxisführung und Praxisorganisation}

Qualitätsmanagement in der Praxis oder dem MVZ - was alles dazu gehört, wird auf den folgenden zehn Seiten näher erläutert. Dabei stehen zwei Bereiche im Mittelpunkt: Führung und Organisation sowie Patientenversorgung und Patientenperspektive. Für jeden Bereich werden geeignete Mittel und Aktivitäten - sogenannte Instrumente vorgestellt. Sie helfen, die Abläufe reibungsloser zu gestalten, die Leistungsfähigkeit der Praxis zu steigern und die Zufriedenheit aller Beteiligten zu erhöhen. Dabei wirken die Instrumente Hand in Hand und sind nicht nur in einem Bereich anzuwenden.

Ärzte und Psychotherapeuten wissen, wie Patienten ambulant behandelt und medizinisch versorgt werden - sie tun dies tagtäglich mit ihrem qualifizierten Team. Um eine Praxis oder ein MVZ zu managen, also zu organisieren und zu leiten, braucht es noch mehr als medizinisches Wissen.
Ein gutes QualitätsmanagementSystem ist auf die individuelle Praxissituation zugeschnitten und unterstützt dabei mit Instrumenten, die Praxis zu führen und zu entwickeln. Viele dieser Instrumente werden schon lange als Teil guter Arbeitsorganisation genutzt.
QM-INSTRUMENTE UND PRAXISORGANISATION:

PROZESS- UND ABLAUFBESCHREIBUNGEN, CHECKLISTEN

Zum Beispiel zu:

Notfallmanagement

Hygienemanagement

Kommunikation

Kooperation

Risiko- und Fehlermanagement

Gerätemanagement

Datenschutz und Datensicherheit

REGELUNG VON

VERANTWORTLICHKEITEN

\section{TEAMBESPRECHUNGEN}

MITARBEITERGESPRÄCHE UND -BEFRAGUNGEN

FORTBILDUNGEN, SCHULUNGEN, UNTERWEISUNGEN DES TEAMS
ALLES IM BLICK:

DIE QUALITÄTSMANAGEMENT-KOORDINATORIN

Die Verantwortung für Qualitätsmanagement liegt bei der Leitung der Praxis oder des MVZ, die auch die entsprechenden zeitlichen und finanziellen Ressourcen zur Verfügung stellt. Es empfiehlt sich jedoch eine Mitarbeiterin (oder einen Mitarbeiter) zu benennen, die für das interne QM zuständig ist: Die Qualitätsmanagement-Koordinatorin oder auch QualitätsmanagementBeauftragte. Sie ist beauftragt, das QM-System zu koordinieren. Sie sorgt beispielsweise dafür, dass das QM-Handbuch kontinuierlich aktualisiert wird und behält wesentliche Termine und Aufgaben im Blick. Die Qualitätsmanagement-Koordinatorin sollte geschult sein - sinnvoll ist es, mindestens eine QM-Fortbildung zu besuchen, in der alle relevanten QM-Instrumente und -Methoden vermittelt werden. Die Qualitätsmanagement-Koordinatorin sollte die Kollegen in den gesamten Prozess systematisch einbinden, immer wieder für QM motivieren und kann Aufgaben an das Team delegieren. 


\section{Mein PraxisCheck Hygiene: KOSTENFREIER ONLINE-TEST}

Erfüllen Sie die Anforderungen zur Hygiene in der Praxis? Hat Ihre

Praxis einen vollständigen und aktuellen Hygieneplan? Wie werden medizinische Instrumente gereinigt, desinfiziert und sterilisiert? -

Der kostenfreie Online-Test „Mein PraxisCheck Hygiene“ der KBV

hilft bei einer ersten Übersicht. Mit 19 Fragen zur Hygiene und den entsprechenden Hinweisen zur konkreten Umsetzung erhalten Sie Tipps und weiterführende Empfehlungen.
DAS ONLINE-TOOL

"Mein PraxisCheck"

GIBT ES AUCH ZUR INFORMATIONS-

SICHERHEIT UND ZUM IMPFEN:

www.kbv.de, Suchbegriff

"Mein PraxisCheck"

\section{$\rightarrow$ PROZESS- UND ABLAUFBESCHREIBUNGEN, CHECKLISTEN}

In Prozess- und Ablaufbeschreibungen wird das einheitliche Vorgehen bei der Durchführung von Arbeitsabläufen schriftlich festgelegt. Dies kann personen- beziehungsweise arbeitsplatzbezogen oder auch übergreifend erfolgen. Das Ziel hierbei ist, dass die Tätigkeiten von allen Mitarbeitern einheitlich ausgeführt werden und nachvollziehbar sind. Insbesondere für alle sicherheitsrelevanten Prozesse müssen die wichtigsten Arbeitsschritte aufgelistet und die Verantwortlichkeiten eindeutig geregelt werden.

Checklisten helfen, den Überblick zu behalten und nichts zu vergessen. So kann beispielsweise die Ausstattung des Notfallkoffers anhand einer Checkliste überprüft werden. Ziel des Einsatzes von Checklisten ist es, bei sich wiederholenden Arbeiten eine vollständige und vergleichbare Ausführung zu gewährleisten. Anhand einer Checkliste können ausgeführte Tätigkeiten und erfüllte Aufgaben als erledigt abgehakt werden.

\section{SO UNTERSTÜTZT QEP}

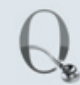

QEP fasst Verfahrensanweisungen und Arbeitsanweisungen unter dem Oberbegriff Ablaufbeschreibungen zusammen. Die Dokumentation der Ablaufbeschreibungen kann in Form von Tabellen oder grafisch visualisiert als Flussdiagramm erfolgen.

\section{BEISPIEL}

\section{NOTFALLMANAGEMENT}

Im Notfall muss alles schnell gehen. Da ist wichtig, dass jeder Handgriff sitzt. Die systematische Vorbereitung auf Notfallsituationen und die kontinuierliche Fortbildung im Team sind wichtige Elemente eines guten Notfallmanagements. Ist klar geregelt, wann die Praxismitarbeiter den Arzt oder Psychotherapeuten einschalten? Sind die Medikamente im Notfallkoffer aktuell und vollständig? Wie erkennen Mitarbeiter am Telefon einen Notfall? Das sind Fragen, die geklärt sein sollten.

\section{SO UNTERSTÜTZT QEP}

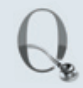

Schulungen sollten möglichst jährlich - je nach Leistungsspektrum und Patientenklientel - durchgeführt und mit praktischen Übungen trainiert werden. Zum Notfallmanagement einer jeden Praxis gehört außerdem eine angemessene, vollständige und funktionsfähige Notfallausstattung.

\section{BEISPIEL}

\section{HYGIENEMANAGEMENT}

Hygiene ist für Praxen und MVZ selbstverständlich. Sie ist ein Muss, um Patienten und Mitarbeiter vor Infektionen zu schützen und die Verbreitung von gefährlichen Keimen zu verhindern.

\section{SO UNTERSTÜTZT QEP}

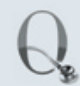

Jeder Praxisinhaber ist verpflichtet, ein Hygienemanagement zu etablieren. So sind beispielsweise Maßnahmen zur Desinfektion, Reinigung und Sterilisation von Geräten schriftlich in einem Hygieneplan festzulegen. QEP zeigt, was alles dazugehört und bietet verschiedene Mustervorlagen, zum Bespiel für einen Reinigungs- und Desinfektionsplan. Mit dem kostenfreien Online-Test „Mein PraxisCheck“ können Praxen in wenigen Minuten überprüfen, wie gut ihr Hygienemanagement ist.

\section{MEHR INFORMATIONEN}

$\lambda$ Leitfaden zur Hygiene des Kompetenzzentrums Hygiene und Medizinprodukte der KVen und der KBV:

www.hygiene-medizinprodukte.de/download

$\lambda$ Schulungsmaterial der Aktion Saubere Hände zur Händehygiene:

www.aktion-sauberehaende.de

$\pi$ MRSA-Information der KBV:

www.kbv.de, Suchbegriff „MRSA“ 


\section{BEISPIEL}

\section{RISIKO- UND FEHLERMANAGEMENT}

Risiken zu vermeiden und aus Fehlern zu lernen spielt für die Patientensicherheit eine wichtige Rolle. Ein entscheidender Punkt dabei ist, wie Ärzte und Psychotherapeuten mit Fehlern umgehen und was sie tun, damit erst gar nichts passiert. QEP hilft dabei, risikobehaftete Abläufe möglichst sicher zu gestalten. Dazu gehört in der Patientenversorgung besonders die Arzneimitteltherapie. Um dort Fehler zu vermeiden, sollten Patienten mithilfe eines Medikationsplanes stets über die richtige Einnahme informiert werden. Größte Sorgfalt ist zudem bei der Zuordnung und Beschriftung von Laborproben und Befunden geboten. $\mathrm{Zu}$ den besonders sicherheitsrelevanten Prozessen zählen ferner alle invasivdiagnostischen und -therapeutischen Maßnahmen, das Erkennen von Notfallsituationen und das Einschätzen der Dringlichkeit bei der Terminvergabe. Weitere Beispiele sind, dass bei Diagnosestellung alle aktuellen Befunde vorliegen und vor therapeutischen Maßnahmen eine umfassende Aufklärung erfolgt.

Mit Fehlern offen umgehen

Praxisintern sollte es eine Möglichkeit geben, über Fehler zu sprechen, um daraus zu lernen und dieselben Fehler nicht zweimal zu machen. Es geht nicht darum, wer etwas falsch gemacht hat, sondern was die Ursache gewesen ist.

\section{SO UNTERSTÜTZT QEP}

Das Thema Patientensicherheit ist im gesamten QEP-QualitätszielKatalog verankert, insbesondere in Kapitel 2 „Patientenrechte und Patientensicherheit“. QEP beinhaltet konkrete Vorschläge zur Organisation von Abläufen, zur Regelung von Verantwortlichkeiten sowie zum Umgang mit Fehlern. Im QEP-Manual gibt es ein Formblatt zur Fehler- und Ergebnisdokumentation.

\section{MEHR INFORMATIONEN}

$\nearrow$ Das QZ-Modul Patientensicherheit steht im sicheren Netz der KVen (SNK) zum Download zur Verfügung. $\pi$ QEP-Newsletter zu Risiko- und Fehlermanagement Nr. 21/2015: www.kbv.de, Suchbegriff "QEP-Newsletter"

$\pi$ KBV-Themenseite: www.kbv.de, Suchbegriff "Fehlermanagement" $\lambda$ Musterdokumente aus dem QEP-Manual: Verantwortlichkeitsplan: www.kbv.de, Suchbegriff „Verantwortlichkeitsplan" Organigramm: www.kbv.de, Suchbegriff „QEP-Organigramm“

\section{FEHLERBERICHTS- UND LERNSYSTEME}

Eine Möglichkeit, Fehler auch außerhalb der eigenen Praxis zu kommunizieren, sind Berichts- und Lernsysteme im Internet. Sie zielen darauf ab, dass möglichst viele Beteiligte aus den anonym gemeldeten Fehlern anderer lernen.

$\pi$ CIRSMEDICAL: CIRS steht für „Critical Incident Reporting-System“. Es richtet sich an Vertrags- und Krankenhausärzte. Betreiber: Das Ärztliche Zentrum für Qualität in der Medizin (ÄZQ) im Auftrag von KBV und Bundesärztekammer. www.patientensicherheit-online.de

$\lambda$ JEDER FEHLER ZÄHLT: Das Fehlerberichts- und Lernsystem ,Jeder Fehler zählt“ richtet sich in erster Linie an hausärztliche Praxen. Betreiber: Institut für Allgemeinmedizin der Johann Wolfgang Goethe-Universität in Frankfurt am Main in Kooperation mit der Techniker Krankenkasse. www.jeder-fehler-zaehlt.de

\section{REGELUNG VON} VERANTWORTLICHKEITEN

Werden Praxen nach den Vorteilen eines Qualitätsmanagements gefragt, sagen viele, dass endlich klar sei, wer wofür verantwortlich ist. Dies gebe allen im Team mehr Sicherheit und spare Zeit.

Für folgende Bereiche ist eine klare Aufgabenverteilung besonders wichtig:

Diagnostische und therapeutische Abläufe

Dokumentation in der Patientenakte

Abrechnung der Leistungen

Hygiene

Arbeitsschutz und -sicherheit

Datenschutz und -sicherheit

Medizinprodukte und Medizingeräte

Strahlenschutz

Gefahrstoffe

Bestellungen und Einkauf

Es empfiehlt sich, zunächst die Aufgaben zu definieren und dann gemeinsam im Team festzulegen, wer wofür zuständig und verantwortlich ist. Nach Möglichkeit sollte immer eine Vertretung benannt werden. In einer Matrix oder einer Tabelle werden die Verantwortlichkeiten dokumentiert und übersichtlich dargestellt. Auch ein Organigramm kann sinnvoll und nützlich sein.

\section{SO UNTERSTÜTZT QEP}

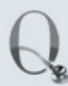

Um die gesetzlichen Anforderungen, beispielsweise zu Hygiene, Datenschutz, Arbeitsschutz oder Medizinprodukten zu kennen und zu erfüllen, müssen die Mitarbeiter bestimmte Kenntnisse besitzen oder Fortbildungen besucht haben. Hierzu finden Sie im Kapitel 4 „Führung und Organisation“ des QEP-Qualitätsziel-Katalogs ausführliche Hinweise und weiterführende Informationen. 
Teambesprechungen zählen zu den bekanntesten und am häufigsten genutzten QM-Instrumenten. Ohne einen regelmäßigen Austausch kann kein Team miteinander arbeiten. Besprechungen sind wichtig, damit alle Mitarbeiter auf dem

\section{VORSCHLAG ZUR ORGANISATION VON TEAMBESPRECHUNGEN}

\begin{tabular}{|c|c|}
\hline & $\begin{array}{l}\text { Es wird ein regelmäßiger Termin fest im Praxiskalender eingeplant. } \\
\text { Empfehlung: mindestens einmal im Monat. Dauer: } 1 \text { bis } 1,5 \text { Stunden. }\end{array}$ \\
\hline TIP & $\begin{array}{l}\text { Nicht länger, da sonst die Aufmerksamkeit und Beteiligung nachlässt. } \\
\text { Lieber öfter und kürzer. }\end{array}$ \\
\hline & Es gibt eine Tagesordnung mit den Besprechungsthemen. \\
\hline TIP & $\begin{array}{l}\text { Die Themen werden im Vorfeld gesammelt, zum Beispiel mit Aushang } \\
\text { im Aufenthaltsraum. }\end{array}$ \\
\hline & $\begin{array}{l}\text { Für die Vorbereitung sorgt eine Person, beispielsweise die Qualitätsmanage- } \\
\text { ment-Koordinatorin. Es gibt einen Moderator und einen Protokollanten. }\end{array}$ \\
\hline TIP & $\begin{array}{l}\text { Tauschen Sie immer mal wieder die Rollen. Jeder im Team kann die } \\
\text { Sitzungen im Wechsel moderieren oder das Protokoll schreiben. }\end{array}$ \\
\hline & $\begin{array}{l}\text { Die Ergebnisse der Teamsitzung werden im Protokoll oder/und direkt } \\
\text { in einem Zeit- und Maßnahmenplan mit Verantwortlichkeiten und } \\
\text { Termin dokumentiert. }\end{array}$ \\
\hline & $\begin{array}{l}\text { Eine Teilnehmerliste liegt aus. Dort unterschreibt jeder Mitarbeiter, der } \\
\text { anwesend ist. Diese Liste und das Protokoll dienen dem Nachweis, wer } \\
\text { anwesend war, was konkret besprochen wurde, was neu eingeführt wurde } \\
\text { oder wer wofür verantwortlich ist. }\end{array}$ \\
\hline TIP & $\begin{array}{l}\text { Alle Mitarbeiter, die bei der Sitzung nicht dabei waren, sollen die besprochenen } \\
\text { Inhalte zur Kenntnis nehmen und mit Handzeichen/Kürzel bestätigen. }\end{array}$ \\
\hline & $\begin{array}{l}\text { Die Qualitätsmanagement-Koordinatorin (oder eine andere festgelegte } \\
\text { Person) überprüft in regelmäßigen Abständen - spätestens bis zur nächsten } \\
\text { Teamsitzung - , ob die Aufgaben bearbeitet wurden bzw. was bis zu dem } \\
\text { Zeitpunkt noch nicht erledigt wurde. }\end{array}$ \\
\hline TIPP & $\begin{array}{l}\text { Aufgaben, die noch offen sind, werden auf die Tagesordnung der nächsten } \\
\text { Sitzung gesetzt und erneut besprochen. }\end{array}$ \\
\hline
\end{tabular}

\section{MEHR INFORMATIONEN}

त Musterdokument aus dem QEP-Manual:

Teamprotokoll:

www.kbv.de, Suchbegriff "QEP-Teamprotokoll“ gleichen Stand sind, auch wenn sie beispielsweise aufgrund von Teilzeit nicht ganztägig vor Ort sind. Frequenz und Umsetzung hängen von Teamgröße und Leistungsspektrum ab.

Mitarbeitergespräche zwischen Führungskraft und Mitarbeiter dienen dazu, strukturiert spezifische Inhalte zu besprechen. Eine gute Vorbereitung sowie eine ruhige Atmosphäre und ausreichend Zeit sind wichtig. Gesprächsinhalte können Leistungsbeurteilungen, Weiterbildungswünsche, Entwicklungsmöglichkeiten und offene Fragen sein. Es können auch Zielvereinbarungen geschlossen werden. Die Gespräche sollten mithilfe von Formblättern, Leitfäden oder Checklisten geführt werden, die gleichzeitig die Struktur für die Gesprächsführung vorgeben und als Dokument für die Personalakte dienen.

Mitarbeiterbefragungen liefern der Praxisleitung Anregungen für Veränderungen und Verbesserungspotentiale. Je nach Größe der Praxis oder des MVZ können sie mündlich oder schriftlich durchgeführt werden. Alternativ stehen verschiedene Fragebögen zum Teil kostenlos zur Verfügung. Ein Beispiel dazu finden Sie im QEP-Manual.

\section{FORTBILDUNGEN, SCHULUNGEN, UNTER- WEISUNGEN DES TEAMS}

Gut qualifizierte Mitarbeiter sind in der Regel motivierter, übernehmen Verantwortung und machen weniger Fehler. Neben den verpflichtenden Unterweisungen des Teams, beispielsweise zur Hygiene, können regelmäßige Schulungen und Fortbildungen der Mitarbeiter zu einem stabilen und langfristigen Arbeitsverhältnis beitragen. Eine Grundlage zur systematischen Planung von Fortbildungen können die Mitarbeitergespräche oder die besprochenen Themen in den Teamsitzungen sein.

\section{TIPP} gen bieten ein umfangreiches Fortbildungsprogramm an. Sie finden die Angebote auf der Internetseite Ihrer KV. 


\section{QM sorgt für Sicherheit: Patientenversorgung}

Es darf nichts schiefgehen:

Die medizinische Versorgung der

Patienten ist das $A$ und $O$ jeder

Praxis. Hohe Qualität erfordert nicht nur hohes Fachwissen und Können. Strukturierte Abläufe tragen ebenfalls zum Behandlungserfolg bei und sorgen dafür, dass die Patienten zufrieden sind. QM leistet auch hierbei Hilfestellung.

QM UND PATIENTENVERSORGUNG:
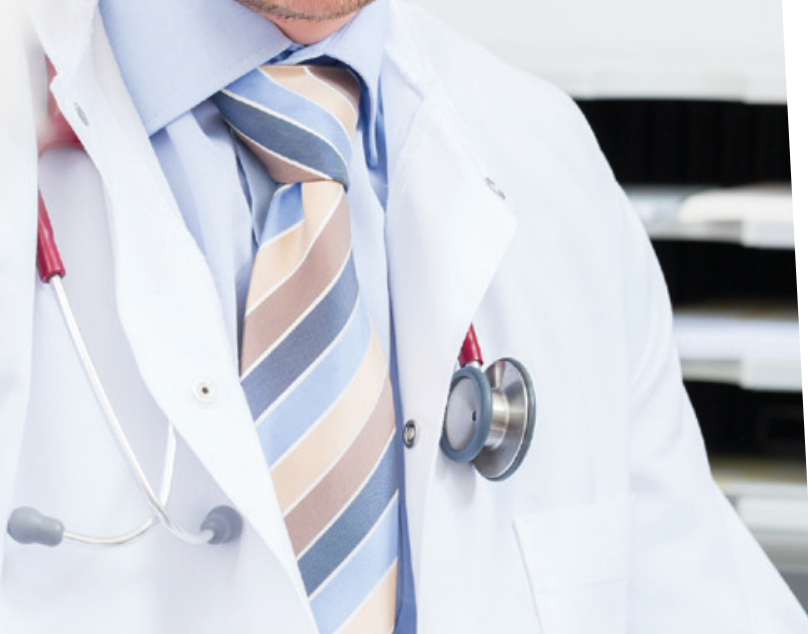
$\cos$
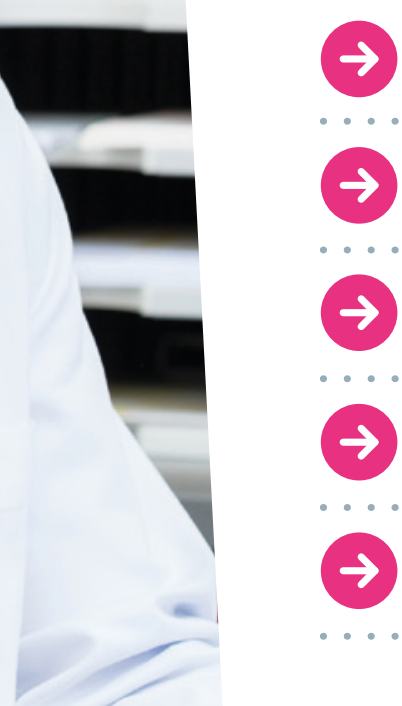

PATIENTENBEFRAGUNG

BESCHWERDEMANAGEMENT

PATIENTENINFORMATION UND AUFKLÄRUNG

BEHANDLUNGSPFADE

UND LEITLINIEN

\section{SCHNITTSTELLENMANAGEMENT}

\section{AMBULANTE QUALITÄTSINDIKATOREN UND KENNZAHLEN - AQUIK ${ }^{\circledR}$}

Das AQUIK-Set ist ein Satz valider, transparenter Qualitätsindikatoren und Kennzahlen für die vertragsärztliche Versorgung. Es wurde zur Qualitätsförderung und -darstellung entwickelt. Das Set umfasst 48 sowohl fachgruppenübergreifende als auch fachgruppenspezifische Indikatoren für die ambulante Versorgung, beispielsweise zu arterieller Hypertonie, Herzinsuffizienz, Arthrose, rheumatoider Arthritis, gynäkologischen Themen, Impfen, Kreuzschmerz und ADHS. 
"Ihre Meinung ist uns wichtig“ - in Restaurants oder Hotels finden sich häufig Kärtchen mit dieser Aufschrift. Es geht um das Feedback der Besucher. Auch viele Ärzte und Psychotherapeuten nutzen dieses Mittel, gibt es ihnen doch wertvolle Anhaltspunkte, wie zufrieden ihre Patienten wirklich sind.

Patientenbefragungen sind im Rahmen des Qualitätsmanagements sinnvoll, da die Praxen Anregungen für mögliche Verbesserungen erhalten. Und die Patienten erhalten das Signal, dass ihre Wünsche und Kritik ernstgenommen werden. Je nach Art und Umfang der Patientenkontakte können die Befragungen jährlich durchgeführt werden. Um Veränderungen messen und darstellen zu können, sollten systematische Befragungen mindestens alle drei Jahre erfolgen.

\section{SO UNTERSTÜTZT QEP}

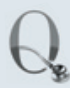

QEP gibt Tipps zur praktischen Durchführung von Patientenbefragungen und bietet dazu verschiedene Musterdokumente. Praxen und MVZ können den kostenlosen Fragebogen zur „Zufriedenheit mit der ambulanten Versorgung - Qualität aus Patientenperspektive“ (ZAP) nutzen.

\section{ZAP-FRAGEBOGEN IN SIEBEN SPRACHEN}

Der Fragebogen zur „Zufriedenheit mit der ambulanten Versorgung - Qualität aus Patientenperspektive" (ZAP) wurde von einer Expertengruppe der Medizinischen Hochschule Hannover entwickelt. Er ist für Patienten gedacht, die bereits mindestens zweimal die Praxis besucht haben. Sie sollen die Bereiche Arzt-Patient-Interaktion, Information, fachliche Kompetenz und Organisation bewerten. Den ZAP-Fragebogen gibt es in sechs weiteren Sprachen: Arabisch, Englisch, Französisch, Russisch, Spanisch und Türkisch. So können auch Patienten befragt werden, die sich in ihrer Muttersprache sicherer fühlen.

\section{VORSCHLÄGE FÜR IHRE PATIENTENBEFRAGUNG}

$\pi$ Befragen Sie mindestens alle drei Jahre etwa zehn Prozent ihrer Patienten, um aussagekräftige Ergebnisse zu erhalten.

$\pi$ Führen Sie die Erhebung anonym durch und nutzen Sie dafür einen validierten Fragebogen.

$\pi$ Wählen Sie einen Zeitraum aus, beispielsweise zwei Wochen, in denen es voraussichtlich nicht zu hektisch zugeht, und verteilen Sie den Fragebogen an alle Patienten, die in diesem Zeitraum in Ihre Praxis kommen.

$\lambda$ Idealerweise verteilt die Praxisleitung selbst den Fragebogen, das erzeugt in der Regel die höchste Akzeptanz bei den Patienten.

$\lambda$ Zur Rückgabe der Fragebögen bietet sich eine Box im Wartezimmer an. $\pi$ Das Team sollte informiert und eingebunden sein.

\section{MEHR INFORMATIONEN}

$\pi$ Den ZAP-Fragebogen, ein ExcelAuswertungstool, Informationen für Patienten und Praxisteams zur Durchführung einer Patientenbefragung finden Sie unter www.kbv.de, Suchbegriff, „ZAP“
Beschwerden sind unangenehm. Doch sie bieten auch eine Chance, das eine oder andere in der Praxis noch besser zu machen. Mitunter steckt hinter einer Beschwerde gar ein Fehler, der dadurch schnell behoben werden kann. Der Umgang mit Beschwerden hat auch etwas mit Unternehmenskultur zu tun. Wie geht die Praxis damit um? Werden Beschwerden ernst genommen und in den Teamsitzungen angesprochen? Was wird den Patienten als Lösung angeboten, damit sie zufrieden die Praxis verlassen können? Die Praxisleitung übernimmt eine wichtige Vorbildfunktion, damit Beschwerden nicht nur besprochen und aktuelle Probleme behoben, sondern auch die Ursachen analysiert und wenn möglich - beseitigt werden.

\section{SO UNTERSTÜTZT QEP}

Das QEP-Manual bietet Musterdokumente mit konkreten Vorschlägen zum Umgang mit Beschwerden (Interne Regelung) an. Es enthält ferner ein Formblatt zur Dokumentation von „unerwünschten“ Ereignissen. So kann das Team die Ursachen besprechen und dafür Sorge tragen, dass diese - wenn möglich behoben werden.

Quelle: QEP-Manual Version 2010, Deutscher Ärzte Verlag

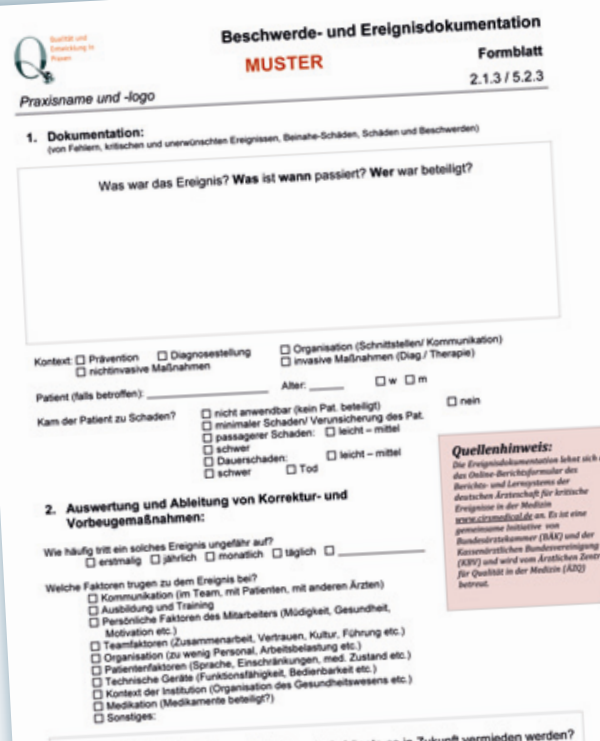


PATIENTENINFORMATION UND AUFKLÄRUNG

Patienten haben viele Fragen. Was bedeutet diese Erkrankung für mich? Welche Behandlung kommt infrage? Welche Rechte habe ich als Patient? Warum muss ich für manche Leistungen selber zahlen? Was kann ich von einer guten Arztpraxis erwarten? Wo finde ich einen Arzt oder einen Psychotherapeuten in meiner Nähe? Die Arzt-Patienten-Kommunikation spielt in der Praxis eine große Rolle. Sie ist wichtig für den Behandlungserfolg. Denn je besser Patienten über eine Therapie informiert sind, desto höher ist deren Adhärenz. Und die Patienten sind insgesamt zufriedener.

Information, Beratung und Aufklärung sowie eine gemeinsame Entscheidungsfindung sind Teil einer guten Behandlung. Ärzte und Psychotherapeuten können hierbei auch auf eine Fülle von Informationsmaterialien zurückgreifen, die es für Patienten in gedruckter Form oder elektronisch im Internet gibt.

\section{TIPP}

Die Kassenärztliche Bundesvereinigung und die Bundesärztekammer stellen über das Ärztliche Zentrum für Qualität (ÄZQ) das Portal www.patienten-information.de mit über 2.000 qualitätsgeprüften Informationen zur Verfügung. Dies sind Publikationen mit leicht verständlichen und verlässlichen Informationen von Berufsverbänden, Fachgesellschaften, Selbsthilfeorganisationen und der ärztlichen Selbstverwaltung. Sie werden regelmäßig gepflegt, aktualisiert und erweitert. Das Portal ist nach Stichworten sortiert von A wie ADHS über Katarakt und Rotaviren bis $\mathrm{Z}$ wie Zöliakie - was eine gezielte Suche einfach macht.

\section{MEHR INFORMATIONEN}

$\pi$ Patienteninformationen der KBV

(Flyer, Merkblätter, Poster):

www.kbv.de/html/3001.php

BEHANDLUNGSPFADE UND LEITLINIEN

Jeder Patient ist anders. Doch so individuell die Behandlung ist, bestimmte Abläufe sind in der Regel immer dieselben. Fachleute sprechen hier von praxisinternen Behandlungspfaden. Darunter sind eine besondere Form von Ablaufbeschreibungen und Checklisten zu verstehen, die sich auf konkrete Erkrankungen, Beschwerdebilder oder Leistungen innerhalb der Praxis beziehen. Solche Behandlungspfade sollten möglichst gemeinsam im Team und mit den klaren Vorgaben durch die Praxisleitung definiert werden, zum Beispiel für Diabetes, Herzinsuffizienz oder Asthma. Im Unterschied zu Leitlinien, die oft von den medizinischen Fachgesellschaften erstellt werden, umfassen Behandlungspfade auch organisatorische und praxisspezifische Aspekte.

Wo es sinnvoll ist, sollten praxis- oder auch einrichtungsübergreifende Behandlungs- beziehungsweise Versorgungspfade erstellt werden. Wann wird an wen überwiesen? Welche Informationen brauchen die weiterbehandelnden Ärzte, Psychotherapeuten oder Physio-, Logo- und Ergotherapeuten? Was benötigen die Patienten und ihre Angehörigen an weiterführenden Informationen? Wie muss die Kommunikation und Kooperation mit dem Krankenhaus oder der Pflegeeinrichtung erfolgen?

\section{SO UNTERSTÜTZT QEP}

QEP zeigt Beispiele von Behandlungspfaden und weist als Hilfe bei der Erstellung auf die besonderen Bedingungen des SGB V und eine Berücksichtigung evidenzbasierter Leitlinien hin.

\section{MEHR INFORMATIONEN}

$\pi$ Leitlinien und Methoden der evidenzbasierten Medizin: www.cochrane.de www.aezq.de www.leitlinien.de www.awmf-online.de www.degam.de www.ebm-netzwerk.de

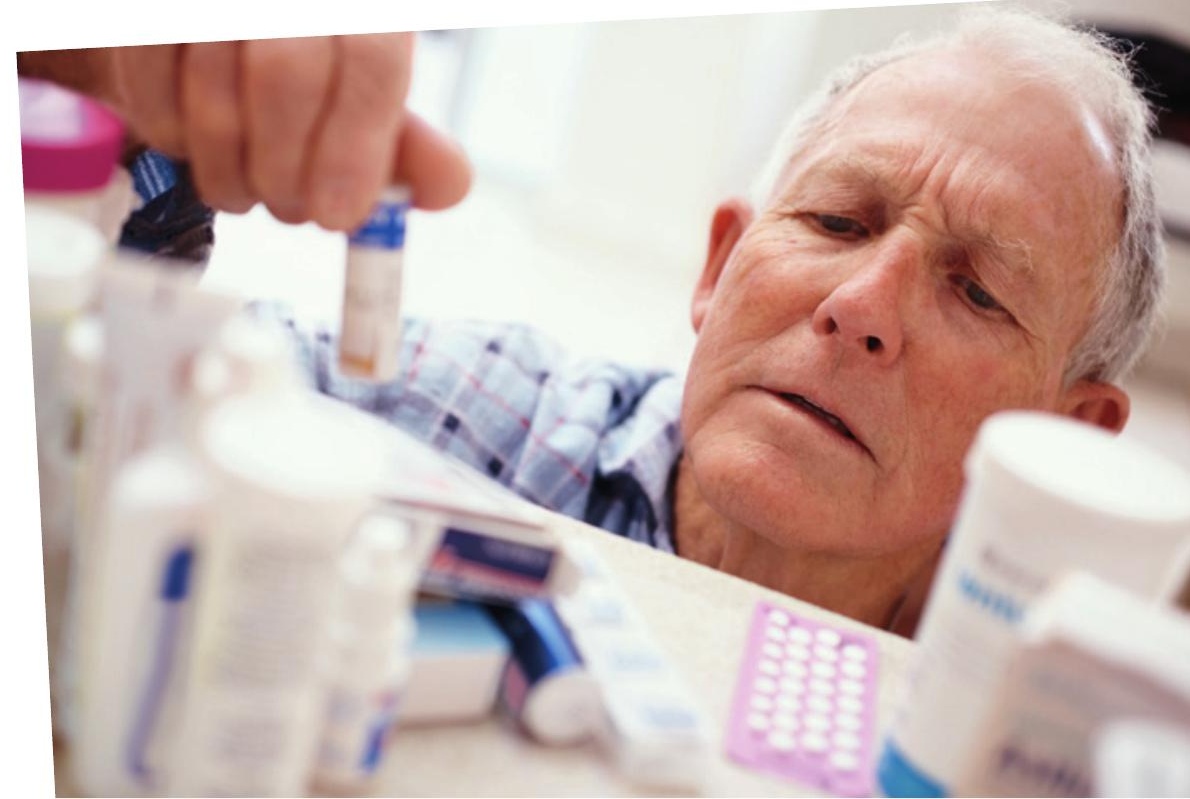




\section{DISEASE-MANAGEMENT-PROGRAMME}

Disease-Management-Programme (DMP) sind strukturierte Behandlungsprogramme für chronisch kranke Menschen. Die Versorgung verläuft nach standardisierten Vorgaben, bei der alle Schritte der Behandlung auf Grundlage evidenzbasierter Leitlinien aufeinander abgestimmt werden. Auch die Schnittstellen zwischen Haus- und Facharzt werden genau definiert. Ein wesentlicher Erfolgsfaktor ist die Mitarbeit der Patienten. Dazu legen Ärzte und Patienten gemeinsam Therapieziele fest und stimmen den Behandlungsverlauf miteinander ab. Patientenschulungen zur Stärkung des Selbstmanagements sind ein fester Bestandteil der Programme.

Zur Qualitätssicherung dokumentieren die Ärzte den Behandlungsverlauf regelmäßig. Die standardisiert erhobenen Daten werden von Krankenkassen und Kassenärztlichen Vereinigungen ausgewertet. Über Feedbackberichte erhalten die Ärzte Informationen über die Versorgung der eigenen Patienten. Gleichzeitig sollen die Berichte dabei helfen, die Qualität der Behandlung zu überprüfen und mit anderen Praxen der jeweiligen KV-Region zu vergleichen. Die Anforderungen an strukturierte Behandlungsprogramme werden vom Gemeinsamen Bundesausschuss beschlossen. Die bundesweiten Vorgaben werden regional in Verträgen zwischen Krankenkassen und Kassenärztlichen Vereinigungen umgesetzt.

Mehr zum Thema DMP: www.kbv.de, Suchbegriff ,DMP“

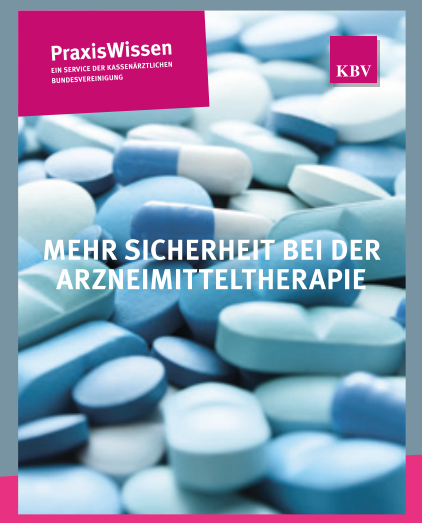

\section{SICHERE ARZNEIMITTELTHERAPIE}

Die Arzneimitteltherapie ist ein sehr wichtiger Bereich der Patientenversorgung, der aber auch mit vielen Risiken behaftet ist. Von der Therapieentscheidung über das Ausstellen des Rezeptes bis hin zur Therapieüberwachung - im gesamten Medikationsprozess können Fehler passieren. Für mehr Sicherheit kann zum Beispiel ein Medikationsplan sorgen. Der Aktionsplan zur Verbesserung der Arzneimitteltherapiesicherheit in Deutschland sieht vor, einen einheitlichen Medikationsplan für Patienten einzuführen. Ein Ziel ist es, den Patienten einen Überblick aller einzunehmenden Arzneimittel zu ermöglichen und Hinweise für deren richtige Anwendung zu geben.
SCHNITTSTELLENMANAGEMENT

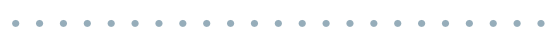

Bei einem Wechsel der Patienten von der Praxis in eine andere Einrichtung, beispielsweise ins Krankenhaus, sind für den Behandlungserfolg nahtlose Übergänge wichtig. Besonders bei der Einweisung und der Entlassung kommt es auf eine enge Abstimmung zwischen ambulant tätigen Ärzten, Psychotherapeuten und Krankenhausärzten an, um eine Kontinuität der Versorgung zu gewährleisten. Hilfreich sind neben einer guten Kommunikation Regelungen zur Überleitung der Patienten von einer Einrichtung in die andere, zum Beispiel zur Arzneimittelversorgung oder zur Weiterführung der Therapie.

Tipps, um eine gute Versorgung beim Wechsel des Patienten in andere Einrichtungen oder nach der Entlassung aus dem Krankenhaus zu gewährleisten, finden Sie in den Checklisten für das ärztliche Schnittstellenmanagement zwischen den Versorgungssektoren.

www.aezq.de, Suchbegriff „Schnittstellenmanagement“

\section{TIPP}

Bestimmte Arzneimittel gelten aufgrund eines erhöhten Risikos für unerwünschte Arzneimittelereignisse wie Stürze, Herzrhythmusstörungen oder Blutungen als potenziell inadäquate Medikation (PIM) bei älteren Patienten. Eine Liste mit Arzneistoffen, die bei älteren Menschen vermieden werden sollten, die sogenannte Priscusliste, finden Sie hier: www.priscus.net

In der Servicebroschüre „Mehr Sicherheit bei der Arzneimitteltherapie“, die die KBV gemeinsam mit der Universität Frankfurt herausgegeben hat, erhalten Ärzte praxisnahe Hinweise und Empfehlungen zur Arzneimitteltherapiesicherheit: www.kbv.de, Suchbegriff „Arzneimitteltherapiesicherheit 


\section{FORTBILDUNG}

Es gehört zum Selbstverständnis von Ärzten und Psychotherapeuten, ihre fachliche Kompetenz fortwährend weiterzuentwickeln. Sie sind dazu gesetzlich verpflichtet, unabhängig davon, ob sie niedergelassen, ermächtigt oder angestellt sind. Dass Ärzten und Psychotherapeuten auch ohne Gesetzesvorgabe eine gute Patientenversorgung am Herzen liegt, zeigen verschiedene freiwillige Initiativen wie Qualitätszirkel oder Peer-Review-Verfahren.

\section{FORTBILDUNGSVERPFLICHTUNG}

Innerhalb eines Fünfjahreszeitraums muss jeder mindestens 250 Fortbildungspunkte, sogenannte CME-Punkte (Continuing Medical Education), gegenüber seiner Kassenärztlichen Vereinigung nachweisen. Das sind etwa 50 Stunden pro Jahr. Über 98 Prozent der Vertragsärzte und Vertragspsychotherapeuten erfüllen diese Vorgabe.

\section{QUALITÄTSZIRKEL}

Qualitätszirkel sind eine etablierte Form der Fortbildung. Ärzte und Psychotherapeuten tauschen sich in moderierten Arbeitskreisen über ihre Arbeit aus, um die eigene Behandlungspraxis zu analysieren und gezielt weiterzuentwickeln.

Qualitätszirkel gelten in der vertragsärztlichen Versorgung seit 20 Jahren als eine anerkannte, auf ärztlicher Eigeninitiative beruhende Methode der Qualitätssicherung. Aktuell sind bundesweit regelmäßig mehr als 9.00o Qualitätszirkel aktiv.

Durch die Teilnahme an einem Qualitätszirkel kommen Ärzte und Psychotherapeuten ihrer gesetzlichen Fortbildungsverpflichtung nach - und zwar selbstbestimmt. Fortbildungspunkte werden über die jeweils zuständige Ärzte- beziehungsweise Psychotherapeutenkammer vergeben. Die KBV und die KVen unterstützen finanziell die regionalen Qualitätszirkel mit der Ausbildung von QualitätszirkelTutoren und Qualitätszirkel-Moderatoren.

\section{So finden Sie einen Qualitätszirkel:}

Informieren Sie sich bei Ihrer Kassenärztlichen Vereinigung über Zirkel in Ihrer Nähe. Auch in vielen Mitglieder-Magazinen der KVen wird das Angebot veröffentlicht.

Sprechen Sie einen Qualitätszirkel-Tutor Ihrer KV an und erkundigen Sie sich nach Zirkeln in Ihrer Nähe. Ansprechpartner unter www.kbv.de/qualitaetszirkel.html

Sie können auch selbst einen Zirkel gründen. Hilfestellung dazu geben Ihnen ein Flyer und ein Video-Clip auf der Website der KBV sowie ein Modul des Handbuchs Qualitätszirkel der KBV.

Alle Informationen zur Anerkennung des Zirkels erhalten Sie ebenfalls bei Ihrer KV.
MEHR INFORMATIONEN
त Flyer - Qualitätszirkel in der ambulanten Versorgung $\nearrow$ Flyer - Wie gründe ich einen Qualitätszirkel: www.kbv.de, Suchbegriff "Qualitaetszirkel“" $\lambda$ Fortbildungsverpflichtung: www.kbv.de, Suchbegriff "Fortbildung“

\section{FÜR QUALITÄTSZIRKEL-MODERATOREN:}

त Handbuch Qualitätszirkel der KBV, 3. Aufl. 2013, ISBN 978-3-7691-3528-2.

Das Handbuch bietet thematische Module und die dazu erforderlichen Moderationsmedien, 


\section{QM: Tipps für Einsteiger}

Sie haben sich neu niedergelassen und möchten Qualitätsmanagement in Ihrer Praxis etablieren - aber wo und wie am besten anfangen? Starten Sie am besten mit einem Thema, bei dem Sie durch Veränderungen schnell spürbare Verbesserungen erzielen können. Denn sichtbare Erfolge sind gerade am Anfang wichtig und steigern die Motivation aller Beteiligten.

Mögliche Startpunkte können Regelungen zur Terminvergabe, zur Dokumentation in der Patientenakte und zu Bestellungen und Lagerhaltung sein.Vorschläge zum besseren Ablauf diagnostischer und therapeutischer Maßnahmen oder die Einführung eines Erinnerungssystems für Vorsorgeuntersuchungen und Impfungen zählen ebenfalls dazu. Und auch das Notfallmanagement sollte auf der To-do-Liste ganz oben stehen. Sie werden schnell merken, wie Ihnen QM im Praxisalltag hilft. Schauen Sie dabei stets auf den gesamten Prozess - also: Wer macht was wie und wann. Und beachten Sie alle damit verbundenen gesetzlichen Anforderungen. Gehen Sie in kleinen Schritten voran.

\section{ENTSCHEIDUNG FÜR QM TREFFEN UND TEAM EINBINDEN}

$\lambda$ Sie legen fest, dass QM eingeführt werden soll und besprechen das mit Ihrem Team.

\section{QM-KOORDINATORIN BENENNEN}

$\lambda$ Übertragen Sie einem Mitarbeiter die Aufgabe, alle Aktivitäten und Maßnahmen des Qualitätsmanagements zu koordinieren.

\section{QM-SYSTEM AUSWÄHLEN}

$\pi$ Sie entscheiden - am besten gemeinsam mit der QM-Koordinatorin mit welcher Struktur oder auf Basis welchen QM-Verfahrens das Qualitätsmanagement in Ihrer Praxis oder Ihrem MVZ aufgebaut werden soll. Informieren Sie sich bei Ihrer Kassenärztlichen Vereinigung und besuchen Sie eine entsprechende Fortbildung, zum Beispiel ein QEP-Einführungsseminar.

\section{IST-STAND CHECKEN}

$\pi$ Überlegen Sie systematisch, mit welchen „Qualitätsinstrumenten“ Sie bereits arbeiten und was Sie wo verbessern können. Achten Sie dabei besonders auf gesetzliche Vorgaben, zum Beispiel zur Hygiene und zum Datenschutz. Wie gut Sie diese bereits umsetzen, können Sie mit dem OnlineTest „Mein PraxisCheck“ prüfen.

\section{ZIELE BESTIMMEN,}

\section{DANN LOSLEGEN}

$\boldsymbol{\lambda}$ Leiten Sie auf Basis dieser Selbstbewertung konkrete Aktivitäten und Maßnahmen für Verbesserungen ab und formulieren Sie dafür Qualitätsziele. Nehmen Sie sich zu Beginn nicht zu viel vor. Vier oder fünf Ziele sind zunächst völlig ausreichend. Übertragen Sie diese in Ihren Zeit- und Maßnahmenplan.

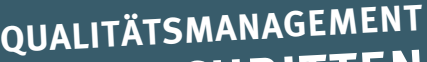 IN 10 SCHRITTEN}

VERANTWORTLICHKEITEN REGELN

$\lambda$ Legen Sie gegebenenfalls weitere Verantwortlichkeiten fest: Beispielsweise für Hygiene, Datenschutz, Arbeitsschutz und -sicherheit, Notfallmanagement und Gefahrstoffe, Medizinprodukte, Strahlenschutz.

\section{PRAXISABLÄUFE FESTLEGEN}

$\pi$ Besprechen Sie im Team Praxisabläufe, vor allem die, die besonders sicherheitsrelevant und/oder fehleranfällig sind. Dazu gehören zum Beispiel sämtliche invasive Tätigkeiten, komplexe diagnostische Untersuchungen und die Verordnung von Arzneimitteln. Je nach Größe des Teams und der Anzahl der beteiligten Mitarbeiter sollten die wichtigsten Abläufe schriftlich festgehalten werden.

\section{OFFEN FÜR VERBESSERUNGEN SEIN} $\pi$ Sprechen Sie im Team offen über Beschwerden, Fehler und das, was nur fast schief gegangen wäre. Akute Probleme müssen sofort geklärt werden, andere haben Zeit bis zur nächsten Teamsitzung. Sammeln Sie möglichst alles, worüber Sie oder andere sich geärgert haben und was nicht wieder vorkommen soll. Nur so kann systematisch nach Ursachen und Verbesserungsmöglichkeiten gesucht werden.

\section{DRAN BLEIBEN}

त Sprechen Sie regelmäßig über Ihre QM-Maßnahmen im Team. Halten Sie Vereinbarungen schriftlich fest, am besten immer verbunden mit einem Verantwortlichen und einem Termin. Und denken Sie daran: Regeln gelten für alle.

\section{UMSETZUNG REGELMÄSSIG ÜBERPRÜFEN}

$\lambda$ Überprüfen Sie die Umsetzung Ihrer Vorhaben, sonst verlaufen diese im Sande. Loben, belohnen und motivieren Sie sich gegenseitig. 
Wer als junger Arzt eine Praxis übernimmt oder völlig neu aufbaut, hat einen riesigen Berg organisatorischer Aufgaben vor sich und kann - besonders in der Anfangsphase - gut und gerne auf zusätzliche Belastungen verzichten. Doch hier lauern Missverständnisse. Wie zum Beispiel bei der Einführung eines gesetzlich vorgeschriebenen Qualitätsmanagements. Das steht in dem weit verbreiteten Ruf unnötiger und zeitraubender bürokratischer Belastung. „Völlig zu Unrecht“, findet die Hamburger Neurologin Dr. Rita Trettin*. Sie plädiert leidenschaftlich dafür, das Qualitätsmanagement als Chance für die Festlegung verbindlicher Arbeitsinhalte und -abläufe zu etablieren - und zwar von Anfang an. Qualitätsmanagement ist für sie der Schlüssel zu einer effektiven und kostensparenden Praxisstruktur.

Die Fachärztin und Ob-Frau der KV in ihrem Bezirk erinnert sich an einen jungen HNO-Kollegen, der ihr sein Leid über die vielen Aufgaben am Anfang seiner Praxis klagte. Das Qualitätsmanagement habe er in dieser Situation als etwas empfunden, „was noch oben drauf kommt“. Dr. Trettin: „Aber wann, wenn nicht gleich zu Beginn einer Praxistätigkeit ist der günstigste Zeitpunkt, sich mit den Mitarbeitern zusammenzusetzen, Pflichten und Aufgaben der Praxis zu definieren, Arbeitsplatzbeschreibungen und -abläufe für alle verbindlich zu formulieren?" Gerade für „Neulinge“ sei Qualitätsmanagement eine Hilfe, um sich im Gestrüpp der vielen Vorschriften und Erfordernisse überhaupt erst einmal zurechtzufinden oder fachliches Potenzial unter den Mitarbeitern zu erkennen und $\mathrm{zu}$ fördern.

\section{ALLE MITARBEITER EINBINDEN}

Für die Ärztin ist entscheidend, alle ihre Mitarbeiterinnen auf diesem Weg mitzunehmen und dem Qualitätsmanagement im Praxisablauf auch genügend Zeit einzuräumen. Verantwortlich für das Qualitätsmanagement ist eine langjährige Mitarbeiterin, doch letztlich ist das gesamte Personal in der Pflicht. Regelmäßige Besprechungen, in denen es nicht nur um aktuelle Aufgaben geht, sondern auch über Fehler diskutiert wird, gehören dazu. In einer eigens geschaffenen Liste kann jeder Fehler und Probleme notieren, die in den Zusammenkünften zur Sprache kommen sollen. „Im Vordergrund stehen dabei die systemische Analyse von und notwendige Schlussfolgerungen aus Fehlern. Es geht nicht um Schuldzuweisungen", betont die Neurologin. Niemand soll das Gefühl haben müssen, in seiner Qualifikation oder gegenüber anderen Mitarbeitern herabgesetzt zu werden.
Alles das ist Bestandteil des Qualitätsmanagements. Und auch hier sieht Dr. Trettin ein ganz entscheidendes Argument für neue Arzt-Kollegen, sich diesen Aufgaben von Anfang an zu widmen. Man sieht im Verlauf der Zeit Stärken und Schwächen deutlicher und findet Anhaltspunkte für die individuelle Mitarbeiterfortbildung oder entdeckt Fähigkeiten und Kompetenzen, die gefördert werden können. Je früher, desto besser. Jedenfalls besteht für die Hamburger Ärztin Personalführung nicht nur aus Dienstplänen, Gehalts- und Urlaubsfragen.

\section{VORTEIL FÜR DIE EINARBEITUNG}

Und auch dies ist für Dr. Trettin eine wichtige Erkenntnis aus dem Qualitätsmanagement: Eine von Anfang an gut strukturierte Praxis bietet für die Einarbeitung neuer Mitarbeiter einen großen Vorteil. Es gibt für alle Arbeitsplätze verbindliche Anweisungen und für alle Abläufe feste Regeln. Das spart Zeit und erleichtert den „Neuen“ die Orientierung. Sie bekommen eine in der Praxis erarbeitete Einarbeitungsmappe und genügend Zeit, sich damit auseinanderzusetzen. Betreut werden sie in der Hamburger Praxis von einer Mitarbeiterin, die speziell für die Einarbeitung von neuen Kollegen geschult ist. Dr. Trettin: „In der Praxis-Startphase ist der Mitarbeiterstamm meist noch nicht sehr stabil. Da ist es bei der Auswahl von Mitarbeitern ein riesiger Vorteil, wenn man die benötigte personelle Qualifikation anhand verbindlicher Praxisstrukturen exakt bestimmen kann.“

* Dr. med. Rita Trettin ist Fachärztin für Neurologie, Fachärztin für Psychiatrie und Psychotherapie und Diplom-Psychologin. Sie beschäftigt derzeit sieben Voll- und Teilzeitmitarbeiterinnen im Hamburger Stadtteil Winterhude. Ihre Praxis ist seit 2012 nach dem QM-System QEP der KBV und der KVen zertifiziert.
Der QEP-Qualitätsziel-Katalog dient zur Orientierung in der Themenvielfalt des Praxis- und Qualitätsmanagements und verschafft einen guten Überblick. Er hilft bei einer systematischen Selbstbewertung. Achten Sie besonders auf die gekennzeichneten Anforderungen der QM-Richtlinie und weiterer gesetzlicher und normativer Vorgaben.

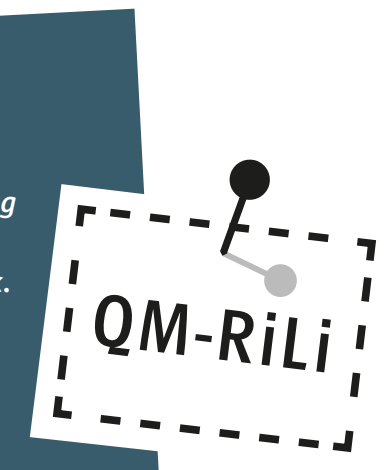




\section{QM in der Psychotherapie}

Auch Psychotherapeuten sind verpflichtet, intern

Qualitätsmanagement aufzubauen und weiterzuentwickeln. Zwar unterscheiden sich die Abläufe von denen einer Arztpraxis allein schon dadurch, dass Psychotherapeuten häufig ohne Personal arbeiten. Doch auch sie profitieren von QM. Denn eine gute Praxisorganisation spart Zeit, gibt Sicherheit und macht die Arbeit leichter.

\section{INDIVIDUELLE ZIELE DEFINIEREN}

QEP ist modulartig aufgebaut. Suchen Sie sich die Ziele und Nachweise aus, die für Ihre Praxis wirklich sinnvoll und umsetzbar sind. Wenn Sie kein Praxisteam haben, können Sie bestimmte Qualitätsziele - beispielsweise zu den Teamsitzungen, Mitarbeitergesprächen und Dienstplänen - einfach überspringen. Folgende Themen sind für alle Praxen relevant:

入 Planung und Vergabe von Terminen $\pi$ Ablauf des Erstgesprächs und weiterer Therapiesitzungen $\pi$ Führen der Patientenakte

$\pi$ Kommunikation mit Kostenträgern wie Krankenkassen

$\pi$ Kooperation mit Ärzten und Gesundheitseinrichtungen

$\pi$ Datenschutz und Datensicherheit

$\pi$ Notfallmanagement

$\pi$ Hygiene-, Brand- und Arbeitsschutz

\section{MUSTERDOKUMENTE ANPASSEN}

Entscheiden Sie selbst, was für Ihre Praxis wichtig ist und passen Sie die Vorschläge von QEP und die Musterdokumente auf Ihre Situation an. Auch muss nicht alles schriftlich geregelt werden - vor allem, wenn Sie keine Mitarbeiter haben.

QEP-MANUAL FÜR PSYCHOTHERAPEUTEN Das QEP-Manual speziell für psychotherapeutische Praxen bietet viele fachspezifische Unterstützungselemente, um intern Qualitätsmanagement aufzubauen und weiterzuentwickeln. Es erscheint Ende 2015. QEP-Manual für Psychotherapeuten vorbestellen bzw. bestellen: www.aerzteverlag.de, Rubrik „Shop“, Suchbegriff „QEP“

\section{QUALITÄT HAT MAN NICHT, QUALITÄT MACHT MAN. WARUM AUCH PSYCHOTHERAPEUTEN QM BENO̊TIGEN. INTERVIEW JULIA BELLABARBA DIPL.-PSYCHOLOGIN UND QEP-TRAINERIN AUS BERLIN}

Oft heißt es, Psychotherapiepraxen seien organisatorisch überschaubar und benötigten kein Qualitätsmanagement. Stimmt das? Diese Meinung höre ich sehr oft und habe viel Verständnis dafür. Aber wir müssen uns darüber klar sein, dass sie auch bedeuten kann: Wer keine Qualität zu bieten hat, der braucht kein Qualitätsmanagement. Das zeigt, dass eine solche Aussage auch problematisch ist. Strukturell mögen die Abläufe in vielen Psychotherapiepraxen nicht sehr kompliziert sein. Aber der Prozess an sich ist sehr komplex: Das Führen eines Patienten in der Psychotherapiepraxis ist sehr differenziert. Alles was ich tue oder nicht tue, hat für ihn eine Bedeutung.

Ein gutes Beispiel ist die Information des Patienten über die beabsichtigte Therapie. Ich kann ihm einen Vordruck vorlegen, den er sich durchlesen und unterschreiben soll. Aber habe ich ihn damit wirklich so informiert, dass er auch verstehen kann, worum es geht? Eine solche Gewissheit kann mir nur das ausführliche Vorgespräch mit ihm bringen, nach dem Motto, ist es das, was Sie sich vorstellen? Als Therapeut trage ich Verantwortung für den Prozess. Und ein Patient spürt, ob ich in kritischen Therapiephasen, wie etwa eine Verschlechterung der Symptomatik während des Therapieverlaufs oder zu seinem Ende hin, angemessen agiere. Das macht für mich einen wesentlichen Teil der Leistungsqualität aus, über die wir hier sprechen.
Und hier kommt Qualitätsmanagement ins Spiel..

QM bietet mir eine gute Hilfestellung bei der Reflexion darüber, wo ich stehe. Das reicht von der Selbsteinschätzung, in welchem Umfang ich fachlich und wissenschaftlich anerkannte Standards im Behandlungsalltag berücksichtige, rechtliche Vorgaben beachte bis hin zu Merkmalen einer qualitätsbezogenen Dokumentation oder externen Kommunikation. Die meisten Kollegen in meinen Seminaren stellen überrascht fest, dass sie viele Qualitätsinhalte bereits in ihrem Praxisalltag leben, und sind dann auch offener für Fragen der Strukturierung. Sie erfahren, dass eine optimierte Praxisstruktur effizient ist, Geld spart und - besonders wichtig - mehr Freiraum für die unmittelbare Arbeit mit den Patienten bringen kann.

Muss Qualitätsmanagement weiterentwickelt werden? Qualität hat man nicht, Qualität macht man. In dieser Feststellung steckt an sich schon die Erkenntnis, dass Qualitätsmanagement ein Prozess ist. Psychotherapeuten mögen hier vergleichsweise einem weniger großen Veränderungsdruck unterworfen sein. Aber wir sollten bedenken, dass wir uns selbst ständig verändern, dass sich eigene Bedürfnisse und Schwerpunkte in der Weiterbildung ändern, dass wir älter werden und so weiter. Das alles kann eigene Ansprüche verändern, hat Einfluss auf die Prozess- und Strukturqualität und erfordert eine Weiterentwicklung des Qualitätsmanagements. 


\section{Auf einen Blick: Das bringt QM}

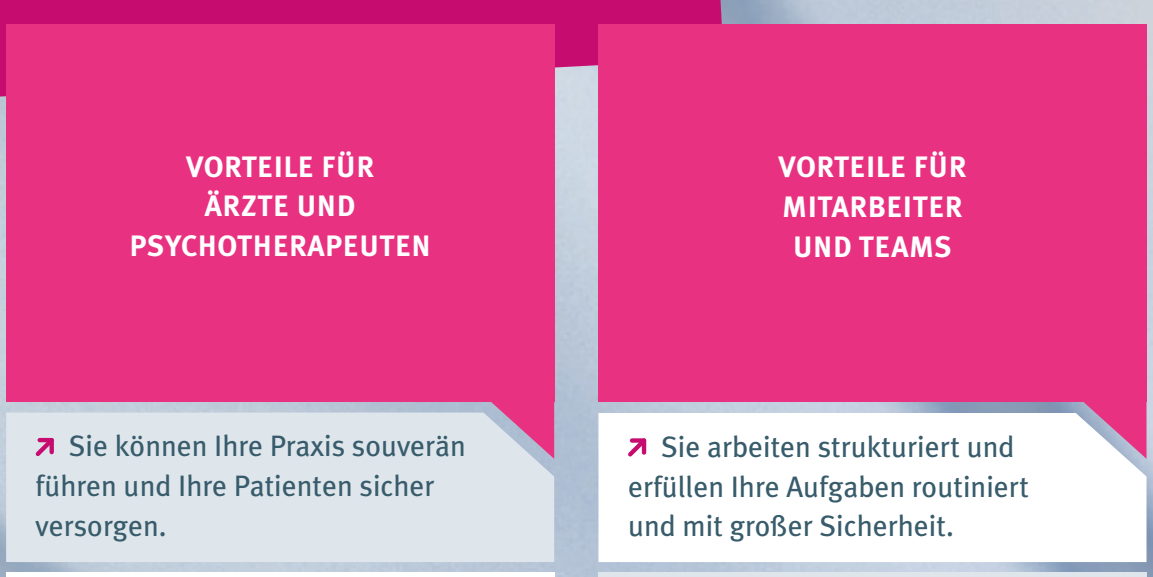

$\boldsymbol{\lambda}$ Ihr strukturierter Praxisbetrieb reduziert Hektik und Stress.

$\lambda$ Die Arbeitsatmosphäre stimmt, Konflikte lassen sich (leichter) lösen.

$\pi$ Sie haben mehr Zeit und Ruhe für Ihre Patienten.

$\pi$ Sie können Ressourcen besser planen und einsetzen.

$\pi$ Sie sind von Aufgaben, die Mitarbeiter übernehmen können, entlastet.

$\boldsymbol{\lambda}$ Sie können Fehler und haftungsrelevante Risiken vermeiden.

入 Sie können Ihre Finanzen schonen.

$\boldsymbol{\lambda}$ Neue Mitarbeiter und Kollegen arbeiten sich leichter ein.

$\boldsymbol{\lambda}$ Sie profitieren von motivierten und zufriedenen Mitarbeitern. $\pi$ Sie wissen, wofür Sie verantwortlich sind und wofür jedes andere Teammitglied zuständig ist.

入 Sie sprechen offen über Probleme und Fehler und suchen gemeinsam nach Lösungen.

$\boldsymbol{\lambda}$ Sie entlasten sich gegenseitig bei der Arbeit, weil nicht alle alles machen (müssen).

$\pi$ Sie unterstützen sich gegenseitig und können sich vertreten.

$\pi$ Eindeutige Regeln und transparente Strukturen tragen zu einem guten Arbeitsklima bei.

$\pi$ Sie nutzen die Chance, sich kontinuierlich fortzubilden und zu qualifizieren.

\section{VORTEILE FÜR PATIENTEN}

$\pi$ Sie profitieren von abgestimmten und sicheren Abläufen sowie einer stressfreien Atmosphäre in der Praxis oder im MVZ.

入 Sie werden sowohl bei Anmeldung als auch im Sprechzimmer über alles Wichtige informiert.

$\pi$ Sie fühlen sich gut beraten und sicher behandelt.

入 Ihr Arzt bzw. Psychotherapeut gewinnt durch eine effiziente Praxisorganisation mehr Zeit für Sie.

入 Sie können Probleme ansprechen. Ihre Beschwerden werden ernst genommen.

$\boldsymbol{\lambda}$ Sie können sicher sein, dass Ihre Daten und Informationen vertraulich behandelt werden.

$\pi$ Durch einen geregelten Informationsfluss zu anderen Einrichtungen ist die Kontinuität Ihrer Behandlung gewährleistet. 


\title{
Hinterher ist man immer schlauer.
}

\author{
QUALITÄTSMANAGEMENT \\ IN DER PRAXIS \\ www.kbv.de/qm
}

www.kbv.de/PraxisNachrichten www.kbv.de/kbv2go E-Mail-Newsletter der Kassenärztlichen Bundesvereinigung, exklusiv für Ärzte und Psychotherapeuten.

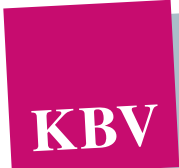

\section{IMPRESSUM}

Herausgeber: Kassenärztliche Bundesvereinigung

Herbert-Lewin-Platz 2, 10623 Berlin

Telefon 030 4005-0, info@kbv.de, www.kbv.de

Redaktion: Dezernat Kommunikation der KBV,

Dezernat Sektorenübergreifende Qualitätsförderung,

Versorgungskonzepte, Qualitätsmanagement,

Patientensicherheit der KBV,

Reportage und Interviewbeiträge auf

den Seiten 4-5, 19, 20: Reinhold Schlitt

Gestaltung: www.malzwei.de

Druck: www.laser-line.de

Fotos: () Dipl.-Psych. Julia Bellabarba;

(C) Fotolia.com: candybox images, contrastwerkstatt, Kzenon, WavebreakmediaMicro; $\odot$ Gettyimages.com: Hybrid Images (c) Christof Rieken; (๑) Reinhold Schlitt; @ Shotshop.com:danstar, Thomas von Stetten/Werbeagentur exakt; $\odot$ Dr. Rita Trettin Stand: November 2015 